\title{
Application des essais Lefranc à l'évaluation du coefficient d'anisotropie hydraulique des sols aquifères
}

Maurice CASSAN

SA Fondasol Études BP 767

84035 Avignon Cedex 3
La détermination du coefficient d'anisotropie des sols n'a été réalisée jusqu'à présent qu'en laboratoire et, compte teruu des difficultés expérimentales, ce paramètre important n'est pris en compte, dans les projets, qu'à partir de valeurs estimées et non mesurées. Il semble que les essais Lefranc, réalisés in situ, pouvaient très bien se prêter à une évaluation pratique de ce coefficient. En effet, l'influence de l'élancement des cavités sur les résultats de l'essai, nulle en milieu isotrope, devient importante en présence d'anisotropie. On a alors montré qu'en faisant varier cet élancement en un même point d'un massif, on pouvait déterminer le coefficient d'anisotropie moyennant une procédure appropriée qu'on a définie. On a également établi les formules explicitant ce coefficient pour toutes les formes usuelles de cavités Lefranc et donné en annexe les tables numériques permettant le dépouillement des essais. après sa parution.
To date, the evaluation of the anisotropic factor value of soils has been carried out by laboratory test results and, considering the experimental difficulties, this fundamental factor has only been taken into account from estimations but not measurements in project designs.

We have then considered that the estimation of this very factor could be carried out through in situ Lefranc test results. Actually, the influence of the cavity's height on the test results, that in ineffective for an isotropic soil, becomes sensible in case of anisotropic soil conditions.

The purpose of this analysis consists in the evaluation of the anisotropic factor value by quantifying the influence of the cavity's height variation according to an appropriate test procedure described below.

Formulae that clarify this factor for usual shapes relative to the Lefranc tests cavities have been estiablished as well, and numerical tables making the test analysis possible are given in appendix. 


\section{Anisotropie hydraulique des sols}

Parmi toutes les difficultés que l'on rencontre dans les problèmes liés aux écoulements souterrains, qu'il s'agisse de l'évaluation de débits d'épuisement ou du tracé des réseaux d'écoulements particulièrement importants dans les études de stabilité, l'une des principales réside dans l'appréciation de l'anisotropie des sols.

En effet, les terrains sédimentaires sont constitués d'une superposition de couches de perméabilités différentes et comme, à l'origine, ces dépôts se faisaient horizontalement, il est évident que dans un tel milieu, toutes choses égales par ailleurs, les vitesses de percolation de l'eau soient différentes dans un écoulement vertical et dans un écoulement horizontal.

Tout le monde connait les formules classiques qui permettent d'évaluer les coefficients de perméabilité équivalents, horizontal $k_{k}$ et vertical $k_{4}$, d'un milieu formé de n strates, chaque strate étant constituée d'un matériau supposé homogène et isotrope. Plus rigoureusement et plus généralement d'ailleurs, la valeur de $\mathrm{k}_{\mathrm{h}}$ correspond à un écoulement parallèle au plan de stratification et $k$ à un écoulement perpendiculaire à ce plan (Fig. 1).

$\mathrm{Si} \mathrm{H}$ et $\mathrm{k}$ désignent respectivement l'épaisseur et le coefficient de perméabilité de la strate de numéro i, on démontre facilement que l'on a :

$$
\begin{aligned}
& \mathrm{k}_{\mathrm{h}}=\frac{\sum \mathrm{k}_{\mathrm{i}} \mathrm{H}_{\mathrm{i}}}{\sum \mathrm{H}_{\mathrm{i}}} \\
& \mathrm{k}_{\mathrm{v}}=\frac{\sum \mathrm{H}_{\mathrm{i}}}{\sum \frac{\mathrm{H}_{\mathrm{i}}}{\mathrm{k}_{\mathrm{i}}}}
\end{aligned}
$$

On peut définir alors le coefficient d'anisotropie par le rapport :

$$
\alpha=\frac{k_{h}}{k_{v}}=\frac{\sum k_{i} H_{i} \sum \frac{H_{i}}{k_{i}}}{\left(\sum H_{i}\right)^{2}}
$$

Si toutes les strates ont la même épaisseur, on voit immédiatement que l'on a :

$$
\begin{aligned}
& \mathrm{k}_{\mathrm{h}}=\frac{1}{\mathrm{n}} \sum \mathrm{k}_{\mathrm{i}}=\text { moyenne arithmétique de } \mathrm{k}_{\mathrm{i}} \\
& \mathrm{k}_{\mathrm{v}}=\frac{\mathrm{n}}{\sum \frac{1}{\mathrm{k}_{\mathrm{i}}}}=\text { moyenne harmonique des } \mathrm{k}_{\mathrm{i}} \\
& \alpha=\frac{1}{\mathrm{n}^{2}} \sum \mathrm{k}_{i} \sum \frac{1}{\mathrm{k}_{\mathrm{i}}}
\end{aligned}
$$

Supposons, pour fixer les idées, que le milieu ne soit constitué que de deux couches d'égale épaisseur, on a alors :

d'où :

$$
\alpha=\frac{\left(k_{1}+k_{2}\right)^{2}}{4 k_{1} k_{2}}=\frac{\left(k_{1}+k_{2}\right)^{2}}{\left(k_{1}+k_{2}\right)^{2}-\left(k_{1}-k_{2}\right)^{2}}
$$

$$
\alpha=\frac{1}{1-\left(\frac{k_{1}-k_{2}}{k_{1}+k_{2}}\right)^{2}}
$$

On voit donc que $\alpha$ est plus grand que l'unité quelles que soient les valeurs de $k_{1}$ et $k_{2}$. Cette propriété se démontre dans toute sa généralité pour les demières formules (2) et (3). Il en résulte qu'en terrain sédimentaire stratifié, on a toujours :

$$
\mathrm{k}_{\mathrm{h}}>\mathrm{k}_{\mathrm{v}}
$$

Dans le cas où les strates sont suffisamment épaisses pour que l'on puisse, soit y prélever des échantillons intacts, soit, de préférence, y réaliser des essais d'eau in situ, le problème de l'anisotropie est facilement résolu comme on vient de le voir. Mais il faut faire très attention à l'utilisation de ces formules car elles ne sont valables que lorsqu'on est certain que l'eau puisse traverser toutes les couches, ce qui est, par exemple, le cas du pompage dans un bâtardeau dont la fiche par rapport au fond de fouille pénètre profondément dans le milieu. Lorsqu'il n'en est pas ainsi, c'est souvent la première couche ou à la rigueur les deux premières qui régissent l'écoulement.

Il ne faut pas croire que les contrastes de perméabilité significatifs ne concernent que les sols stratifiés constitués d'alternances de couches argileuses ou limoneuses et de dépôts granulaires. Ces contrastes peu-

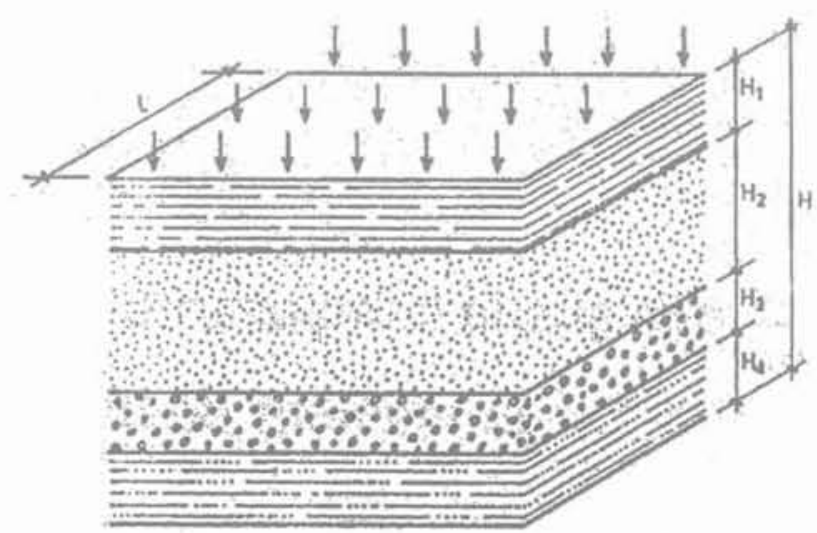

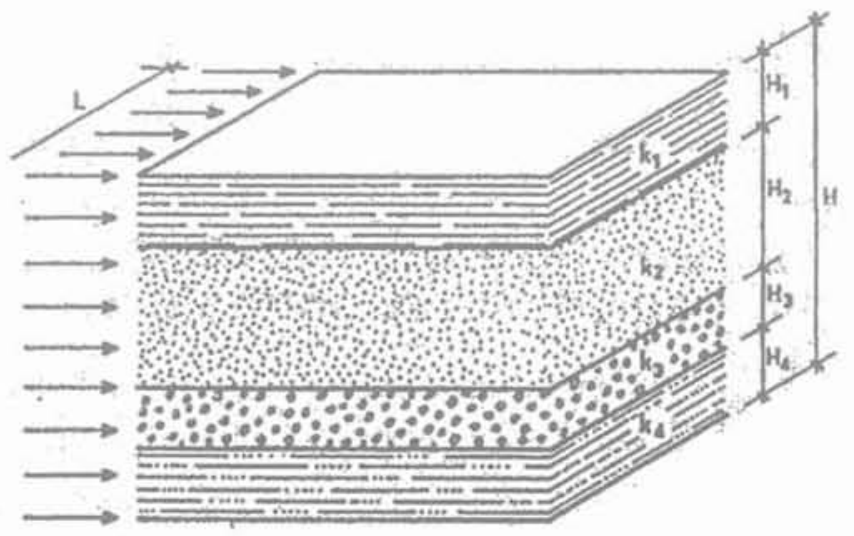

AG.1. Filtration en terrain stratifié. Filtration in stratified soil. 
vent également s'observer dans les sols granulaires eux-mêmes en fonction de leur granulométrie comme on peut s'en rendre compte à partir de la formule de Hazen :

$$
\mathrm{k}(\mathrm{m} / \mathrm{s})=\left(\mathrm{d}_{10} \mathrm{~cm}\right)^{2}
$$

dans laquelle $\mathrm{d}_{10}$ est le diamètre donnant un passant de $10 \%$ sur la courbe granulométrique.

On voit alors que si deux formations granulaires ont des courbes granulométriques décalées d'un module logarithmique, leur perméabilité varie de deux puissances de dix. Sans aller aussi loin dans le contraste, une variation de $d_{10}$ de 1 à 3 entraîne une variation de $k$ de l'ordre d'une puissance de 10. Certes l'utilisation de la formule de Hazen est limitée aux sables dont les diamètres des grains sont compris entre 0,1 et $3 \mathrm{~mm}$ [1] et dont le coefficient d'uniformité $C U=\frac{\mathrm{d}_{60}}{\mathrm{~d}_{10}}$ est inférieur à

2, bien qu'on estime que l'on puisse déplacer cette limite jusqu'à 4 ou 5. Néanmoins, pour des sols granulaires de plus gros diamètre, l'influence de la granulométrie reste importante, ce qui explique que certaines couches de sable peuvent être considérées comme imperméables par rapport à des couches de graviers ou de graves grossières.

Lorsqu'on se trouve en présence d'un sol très finement stratifié constitué par exemple par une alternance de couches d'argiles et de sable d'épaisseur millimétrique, on se rapproche du schéma correspondant aux relations (3), mais il est très difficile de mesurer directement les perméabilités de chaque strate. On ne peut alors que tenter une mesure globale des perméabilités horizontales et verticales équivalentes.

Mais une telle anisotropie existe également dans des formations sédimentaires apparemment homogènes, comme par exemple certains massifs de sables ou de sables et graviers, à cause de leur mise en dépôt par strates successives horizontales. On dit alors que l'on se trouve en présence d'un milieu homogène anisotrope.

Pour les terrains finement stratifiés ou pour les terrains homogènes anisotropes la détermination de $k_{k}$ et de $k_{w}$ peut se faire en laboratoire, mais il ne s'agit là que de mesures ponctuelles dont les valeurs sont peu représentatives du sol en place.

On se propose d'examiner ici dans quelle mesure les essais in situ de type Lefranc pourraient permettre une évaluation plus fiable de $\alpha, k_{n}$ et $k_{v}$.

\section{2}

\section{Essais Lefrane en terrain homogène isotrope}

Rappelons [3] que l'essai Lefranc, qui est régi par la norme Afnor NF-P 94132 (juin 1992), consiste à provoquer la percolation de l'eau à travers la paroi d'une cavité aménagée dans le sol, sous l'effet d'un gradient hydraulique provoqué soit par pompage soit par injection gravitaire d'un débit d'eau constant dans cette cavité (Fig. 2). $\mathrm{Si} B$ et $\mathrm{L}$ désignent respectivement le diamètre et la hauteur de la cavité, on démontre qu'en milieu isotrope la relation qui, à tout instant et a fortiori lorsque le régime permanent est atteint, relie le débit Q pompé ou injecté, d'une part à la charge h génératrice de l'écoulement et d'autre part au coefficient de perméabilité k est :

$$
\mathrm{Q}=\mathrm{m} k \mathrm{~h} \mathrm{~B}
$$
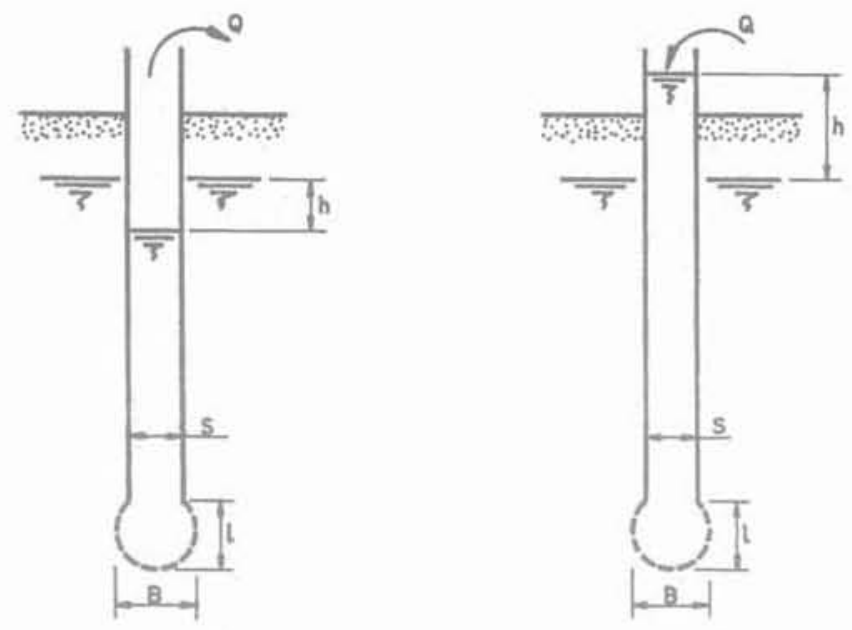

F0.2 Schéma de principe de l'essai Lefranc: a) par pompage ; b) par injection.

Diagram of the Lefranc test : a) by pumping : b) by injection.

où m est un nombre sans dimension appelé coefficient de forme qui n'est fonction que de l'élancement de la cavité défini par le rapport $\lambda=\frac{\mathrm{L}}{\mathrm{B}}$ lequel peut varier de zéro pour le disque limitant le fond du forage, jusqu'à des valeurs aussi grandes que l'on veut.

Le coefficient de forme $m(\lambda)$ de la cavité dans le milieu réel a été explicité analytiquement [3] et son expression dépend de la forme géométrique que l'on peut attribuer à cette cavité.

C'est ainsi que l'on peut répartir les cavités en fonction de l'élancement $\lambda$ en cinq familles géométriques, à chacune desquelles correspond un coefficient de forme différent conformément au tableau I ci-après :

Parmi ces cing familles, la quatrième, c'est-à-dire celle de l'ellipsoìde de révolution aplati, est illusoire car ses coefficients de forme sont inférieurs à 2,44, alors que pour la cavité de hauteur nulle (disque limitant le fond du forage) le coefficient de forme est égal à 2. On voit donc que, lorsque $\lambda$ est inférieur à 0,3 , l'ordre de grandeur des coefficients de forme reste voisin de 2 . On peut alors admettre raisonnablement que l'on se trouve dans le cas du disque circulaire.

La relation (4) et les formules du tableau I, qui sont normatives et qui sont, par conséquent, utilisées en permanence pour évaluer $k$, supposent que le milieu est homogène et isotrope. Or, comme on l'a vu précédemment, l'isotropie est tout à fait exceptionnelle et le cas général est celui des sols anisotropes, même s'ils sont homogènes. Il en résulte que la valeur de $k$ obtenue conformément à la norme, à partir de la relation (4), est une valeur intermédiaire entre $\mathrm{k}_{\mathrm{h}}$ et $\mathrm{k}_{\mathrm{v}}$.

Si, dans une même cavité, on effectue plusieurs essais avec, pour chaque essai, un débit Q différent entraînant par conséquent une valeur de h différente, les rapports Q resteront constants que le sol soit h

isotrope ou anisotrope. En revanche, si on fait varier la hauteur de la cavité et si, pour chaque hauteur, on procède à des essais Lefranc avec, pour chacun d'eux, un débit différent, les valeurs $Q$ varieront d'un essai à h

l'autre, lorsque le terrain est anisotrope, alors qu'elles resteront constantes dans un terrain isotrope. 


\begin{tabular}{l|c|c}
\hline Familles géométriques & Élancements & Coefficient de forme $m(\lambda)$ \\
\hline Ellipsoïde de révolution allongé & $\lambda \geq 1,5$ & $\frac{2 \pi \lambda}{\operatorname{Argsh} \lambda}$ \\
\hline Sphère & $1,5>\lambda>0,7$ & $\pi \sqrt{4 \lambda+1}$ \\
\hline Demi-sphère & $0,7 \geq \lambda>0,3$ & $\pi \sqrt{\frac{4 \lambda+1}{2}}$ \\
\hline Ellipsoïde de révolution aplati & $0,3 \geq \lambda>0$ & $\frac{\pi \sqrt{1-4 \lambda^{2}}}{2 \operatorname{Arccotg}\left(2 \lambda+\sqrt{4 \lambda^{2}+1}\right)}$ \\
\hline Disque circulaire & 0 & 2 \\
\hline
\end{tabular}

\section{3}

\section{Essais Lefranc en terrain homogène anisotrope : évaluation du coefficient d'anisotropie}

\section{1}

\section{Principe de la méthode}

Dans un essai Lefranc réalisé en terrain homogène mais anisotrope, on a bien le sentiment, d'après les considérations précédentes, que plus la longueur de la cavité est importante par rapport à son diamètre, plus la valeur globale de k devra être voisine de $k_{h}$, et qu'inversement pour une hauteur nulle (disque de fond) la valeur de $k$ se rapprochera de $k$. Mais il est faux de conclure hâtivement qu'un essai par le disque de fond de forage permet de mesurer le coefficient de perméabilité verticale et qu'un essai dans une cavité de très grande hauteur permet d'atteindre une valeur convenable de $k_{h}$.

Cette influence du coefficient de forme sur l'évaluation du coefficient de perméabilité équivalent k permet de penser qu'en réalisant deux essais avec des cavités de longueur différentes on devrait pouvoir approcher une valeur tout à fait représentative du coefficient d'anisotropie.

C'est effectivement ce qu'a fait Schneebeli en 1954 [6] mais en se limitant à de faibles élancements (de l'ordre de $\lambda \leq 2$ ) vraisemblablement parce que les expressions analytiques des coefficients de forme étaient, dans ce cas, plus simples. On estime qu'il faut, au contraire, raisonner sur des cavités allongées de façon à pouvoir faire varier le coefficient de forme dans des proportions significatives pour obtenir des résultats suffisamment contrastés.

En effet, les essais Lefranc sont réalisés en général dans des forages dont le diamètre excède rarement $200 \mathrm{~mm}$, la valeur la plus courante étant de l'ordre de $120 \mathrm{~mm}$. Pour ce dernier diamètre, d'après Schneebeli, on devrait faire varier la longueur des cavités entre $12 \mathrm{~cm}$ et $24 \mathrm{~cm}$. Lorsqu'on sait ce qu'est un chantier de forage, on voit bien qu'il est difficile, surtout si la cavité est profonde, de pouvoir connaître l'élancement et, par voie de conséquence, le coefficient de forme avec une précision suffisante. En revanche, si l'on part d'un élan- cement de 4, c'est-à-dire d'une cavité d'environ 0,50 m de longueur et que l'on adopte, pour le deuxième essai, un élancement de 10 , soit 2,5 fois plus, on est conduit à réaliser une cavité de $1,20 \mathrm{~m}$ de hauteur plus facile à définir qu'une cavité de $0,25 \mathrm{~m}$.

Par ailleurs, on peut être très réservé sur la représentativité de l'essai réalisé dans une cavité de hauteur nulle (disque de fond de trou) car une telle cavité est très difficile à réaliser. Si, par exemple, on opère par pompage, on ne sait jamais s'il ne s'est pas produit en fond de trou un phénomène de renard qui, bien que faible, peut altérer la représentativité des mesures.

On montrera plus loin qu'il est hautement souhaitable que les élancements ne descendent pas au-dessous de 1,5 .

Le principe de la méthode consiste à transformer par l'affinité définie dans l'annexe 1, le milieu réel anisotrope en un milieu fictif isotrope, qu'on appellera milieu transformé, et on choisit, dans le cas présent, les rapports d'affinité qui permettent de conserver les distances verticales, c'est-à-dire qu'on utilise les formules [8] de l'annexe 1.

Dans le milieu transformé, homogène et isotrope, l'équation de l'essai Lefranc [4] s'écrit:

$$
Q^{\prime}=m^{\prime} k^{\prime} h^{\prime} B^{\prime}
$$

avec par conséquent, en faisant apparaittre le coefficient d'anisotropie $\alpha$ :

$$
\left[\begin{array}{l}
\mathrm{k}^{\prime}=\mathrm{k}_{\mathrm{v}} \\
\mathrm{B}^{\prime}=\frac{\mathrm{B}}{\sqrt{\alpha}} \\
\mathrm{h}^{\prime}=\mathrm{h} \\
\lambda^{\prime}=\frac{\mathrm{L}^{\prime}}{\mathrm{B}^{\prime}}=\frac{\mathrm{L}}{\mathrm{B}} \sqrt{\alpha}=\lambda \sqrt{\alpha} \\
\mathrm{Q}^{\prime}=\frac{\mathrm{Q}}{\alpha}
\end{array}\right.
$$

Le coefficient de forme m' du milieu transformé n'est fonction que de l'élancement de la cavité dans ce milieu, c'est-à-dire de $\lambda \sqrt{\alpha}$. Or, le coefficient d'anisotropie étant supérieur à l'unité, et cela dans des proportions qui peuvent atteindre des valeurs importantes, l'élancement transformé $\lambda \sqrt{\alpha}$ peut devenir nettement supérieur à l'élancement $\lambda$ de la cavité réelle. Le tableau du paragraphe 2 montre alors que la cavité du milieu 
transformé peut appartenir à une famille géométrique différente de celle de la cavité réelle, et que, par conséquent, les coefficients de forme de ces deux cavités risquent ne pas avoir la même expression mathématique.

Ainsi donc, le coefficient de forme de la cavité transformée n'est pas forcément obtenu en transformant le coefficient de forme de la cavité réelle par l'affinité adoptée. Le seul cas où l'on est certain que les deux cavités réelle et transformée appartiennent à la même famille géométrique est celui des cavités réelles allongées caractérisées par $\lambda>1,5$, car on a alors toujours $\lambda \sqrt{\alpha}>\lambda>1,5$. Dans tous les autres cas, il faudra être très vigilant comme on le verra plus loin.

Dans ce qui suit, on désigne par $m(\lambda \sqrt{\alpha})$ le coefficient de forme de la cavité transformée, sans perdre de vue que pour $\lambda<1,5$, ce coefficient risque de ne pas avoir la même expression mathématique (au facteur $\sqrt{\alpha}$ près) que le coefficient de forme $m(\lambda)$ de la cavité réelle.

L'équation (5) s'écrit alors :

$$
\frac{\mathrm{Q}}{\alpha}=m(\lambda \sqrt{\alpha}) \mathrm{k}_{v} \mathrm{~h} \frac{\mathrm{B}}{\sqrt{\alpha}}
$$

soit encore, puisque $\alpha k_{\mathrm{v}}=\mathrm{k}_{\mathrm{h}}$ :

$$
\mathrm{Q}=\frac{\mathrm{m}(\lambda \sqrt{\alpha})}{\sqrt{\alpha}} \mathrm{k}_{\mathrm{h}} \mathrm{hB}
$$

d'où l'on tire :

$$
k_{h}=\frac{Q \sqrt{\alpha}}{m(\lambda \sqrt{\alpha}) h B}
$$

En comparant les équations (4) et (7) on voit que :

$$
\frac{k_{h}}{k}=\frac{m(\lambda) \cdot \sqrt{\alpha}}{m(\lambda \sqrt{\alpha})}
$$

On constate alors qu'en terrain anisotrope homogène, c'est-à-dire en terrain où $\alpha$, est constant, le coefficient de perméabilité tel qu'on l'évalue de façon réglementaire avec l'essai Lefranc normalisé est fonction de l'élancement de la cavité, ce qui ne peut lui conférer une valeur intrinsèque. Mais il est bien évident qu'en terrain homogène et isotrope, la valeur de k est indépendante de cet élancement. Toutefois, c'est précisément cette influence de la forme de la cavité en terrain anisotrope qui, pour gênante qu'elle puisse paraître, va permettre d'évaluer le coefficient d'anisotropie du sol et, par voie de conséquence, les coefficients de perméabilité horizontale et verticale.

Supposons, en effet, que l'on réalise au même point deux essais Lefranc, le premier avec une cavité d'élancement $\lambda_{1}$ et sous un débit $Q_{1}$, le second avec une cavité d'élancement $\lambda_{2}=n \lambda_{1}$ et sous un débit $Q_{y}$, et que, dans les deux cas le régime permanent ait été atteint. Désignons par $h_{1}$ la hauteur d'eau stabilisée sous le débit $Q_{\text {, }}$ dans le premier essai, et par $h_{\text {, la valeur stabilisée dans }}$ le deuxième essai sous le débit $Q_{2}$.

Si le régime permanent n'est pas atteint dans l'un des deux essais ou dans les deux, on sait que l'interprétation en régime transitoire $[3,4]$ permet d'évaluer pour chacun des essais les coefficients de perméabilité apparents, et, par voie de conséquence de calculer les valeurs de $h_{1}$ et $h_{2}$ correspondant au régime permanent à partir de la relation [4]. Ainsi donc, dans tous les cas, les valeurs de $h_{1}$ et $h_{2}$ sont connues.
On peut alors écrire :

$$
\begin{aligned}
& Q_{1}=\frac{m\left(\lambda_{1} \sqrt{\alpha}\right)}{\sqrt{\alpha}} k_{h} h_{1} B \\
& Q_{2}=\frac{m\left(\lambda_{2} \sqrt{\alpha}\right)}{\sqrt{\alpha}} k_{h} h_{2} B=\frac{m\left(\lambda_{1} \sqrt{\alpha}\right)}{\sqrt{\alpha}} k_{h} h_{2} B
\end{aligned}
$$

d'où l'on tire :

$$
\frac{h_{1} Q_{2}}{h_{2} Q_{1}}=\frac{m\left(n \lambda_{1} \sqrt{\alpha}\right)}{m\left(\lambda_{1} \sqrt{\alpha}\right)}
$$

où, d'après ce qưil a été dit précédemment, $m\left(\lambda_{2} \sqrt{\alpha}\right)$ et $m\left(\lambda_{1} \sqrt{\alpha}\right)$ n'ont pas forcément la même expression mathématique.

Le problème se ramène donc finalement à la résolution d'une équation de la forme :

$$
\mathrm{q}=\mathrm{f}(\mathrm{n}, \mathrm{x})
$$

dans lacuelle :

$$
\mathrm{n}=\frac{\lambda_{2}}{\lambda_{1}} \quad \mathrm{x}=\lambda_{1} \sqrt{\alpha} \quad \text { d'où } \alpha=\left(\frac{\mathrm{x}}{\lambda_{1}}\right)^{2} \text {; }
$$

$\lambda_{1}$ et $\mathrm{n}$ sont connus puisque ce sont les données géométriques des cavités de filtration ;

q est connu puisque ne dépendant que des débits, des variations de charge qui sont des valeurs expérimentales, et éventuellement d'un facteur numérique lié à l'expression du coefficient de forme des cavités.

Comme les expressions analytiques des coefficients de forme sont parfaitement connues (cf. supra) l'équation (9) peut être facilement explicitée et résolue, soit analytiquement dans certains cas, soit numériquement.

Ayant ainsi évalué $\alpha$, les formules (7) ou (8) permettent de calculer le coefficient de perméabilité horizontale.

On remarquera que l'équation fondamentale (9) ne dépend que de l'élancement $\lambda_{i} \sqrt{\alpha}$ des cavités dans le milieu transformé.

\section{2}

\section{Cavités allongées en milieu indéfini}

On a vu que les cavités cylindriques allongées sont celles dont l'élancement $\lambda=\frac{\mathrm{L}}{\mathrm{B}}$ est supérieur à 1,5 aussi bien dans le milieu réel que transformé. On les assimile à des ellipsoîdes de révolution allongés de même axe que le forage et dont les foyers sont les centres des deux cercles qui les limitent.

Leur coefficient de forme a pour expression :

$$
\begin{aligned}
& m(\lambda)=\frac{2 \pi \lambda}{\ln \left(\lambda+\sqrt{\lambda^{2}+1}\right)}=\frac{2 \pi \lambda}{\operatorname{Argsh} \lambda} \\
& m\left(n \lambda_{1} \sqrt{\alpha}\right)=\frac{2 \pi n \lambda_{1} \sqrt{\alpha}}{\operatorname{Argsh}\left(n \lambda_{1} \sqrt{\alpha}\right)} \\
& m\left(\lambda_{1} \sqrt{\alpha}\right)=\frac{2 \pi \lambda_{1} \sqrt{\alpha}}{\operatorname{Argsh}\left(\lambda_{1} \sqrt{\alpha}\right)}
\end{aligned}
$$


d'où : $\frac{m\left(n \lambda_{1} \sqrt{\alpha}\right)}{m\left(\lambda_{1} \sqrt{\alpha}\right)}=\frac{n \operatorname{Argsh}\left(\lambda_{1} \sqrt{\alpha}\right)}{\operatorname{Argsh}\left(n \lambda_{1} \sqrt{\alpha}\right)}$

L'équation (9) s'écrit alors :

$$
\frac{h_{1} Q_{2}}{n h_{2} Q_{1}}=\frac{\operatorname{Argsh}\left(\lambda_{1} \sqrt{\alpha}\right)}{\operatorname{Argsh}\left(n \lambda_{1} \sqrt{\alpha}\right)}
$$

et l'équation (10) prend la forme :

$$
q=\frac{\operatorname{Argsh} x}{\operatorname{Argsh}(n x)}
$$

avec : $\mathrm{q}=\frac{\mathrm{h}_{1} \mathrm{Q}_{2}}{\mathrm{nh}_{2} \mathrm{Q}_{1}}$

L'équation (12) ne peut être résolue que numériquement et l'on pourra utiliser à cet effet les courbes de la figure 3 ou les tables numériques 1 de l'annexe 2.

On en déduit :

$$
\alpha=\left(\frac{x}{\lambda_{1}}\right)^{2}
$$

La relation (8) devient :

$$
\frac{k_{h}}{k_{i}}=\frac{\operatorname{Argsh}\left(\lambda_{i} \sqrt{\alpha}\right)}{\operatorname{Argsh} \lambda_{i}}
$$

et de l'équation (7) on tire :

$$
k_{h}=\frac{Q_{i} \operatorname{Argsh}\left(\lambda_{i} \sqrt{\alpha}\right)}{2 \pi \lambda_{i} h_{j} B}
$$

avec $: i=1$ et 2 .

Les deux valeurs de $k_{h}$ ainsi obtenues doivent être sensiblement égales. S'il en est effectivement ainsi, c'est que le terrain est homogène conformément à I'hypothèse de base. Dans le cas contraire, c'est que le terrain est non seulement anisotrope, mais également hétérogène. Il faut alor's reprendre les essais avec des poches de plus faible hauteur, ce qui n'est pas toujours possible, car l'expérience montre que, souvent, lorsqu'on a fini de dépouiller les essais, le matériel n'est plus sur le chantier !

On sait, par ailleurs, que les essais Lefranc sont souvent perturbés par des phénomènes parasites. C'est pourquol, on estime que dans une procédure d'expérimentation en vue de déterminer le coefficient d'anisotropie, il serait souhaitable de prévoir la réalisation au même point d'au moins trois essais Lefranc avec trois élancements différents, ce qui permettrait d'obtenir trois évaluations de $\alpha$ et de $k_{h}$.

Les courbes représentatives de l'évolution de $\frac{k_{h}}{k}$ en fonction du coefficient d'anisotropie $\alpha$ et de l'élancement de la cavité $\lambda$ (Fig. 4), montrent :

- d'une part que les écarts entre $k_{h}$ et $k$ diminuent lorsque l'élancement augmente et qu'il faudrait un élancement très grand (théoriquement infini) pour que $\mathrm{k}_{\mathrm{h}}=\mathrm{k}$;

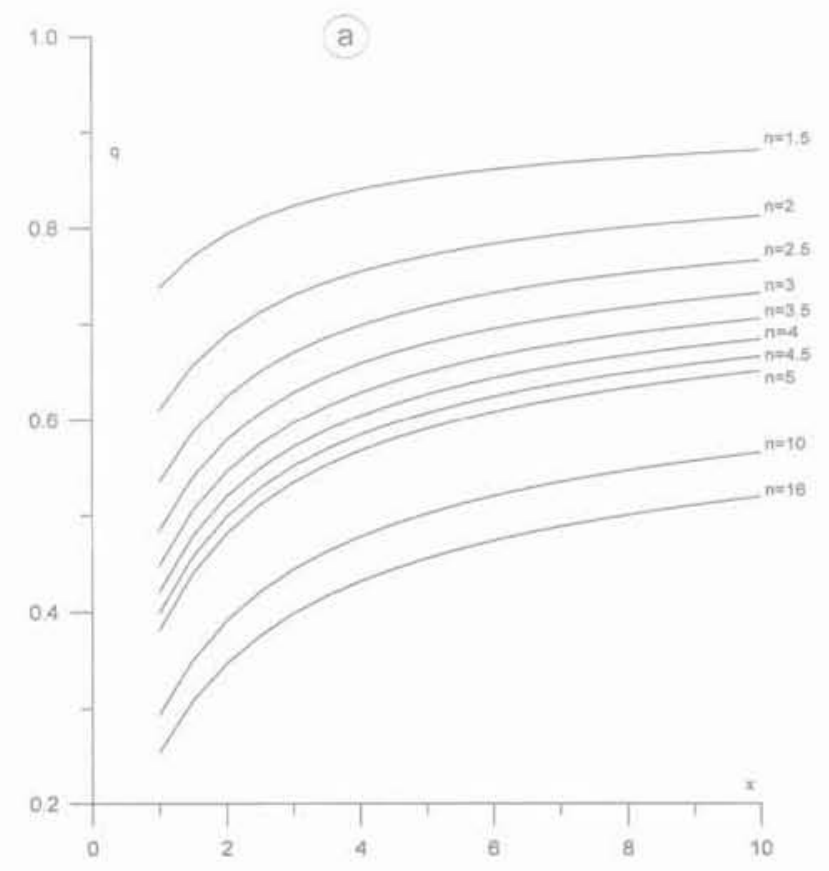

a) $x \leq 10$

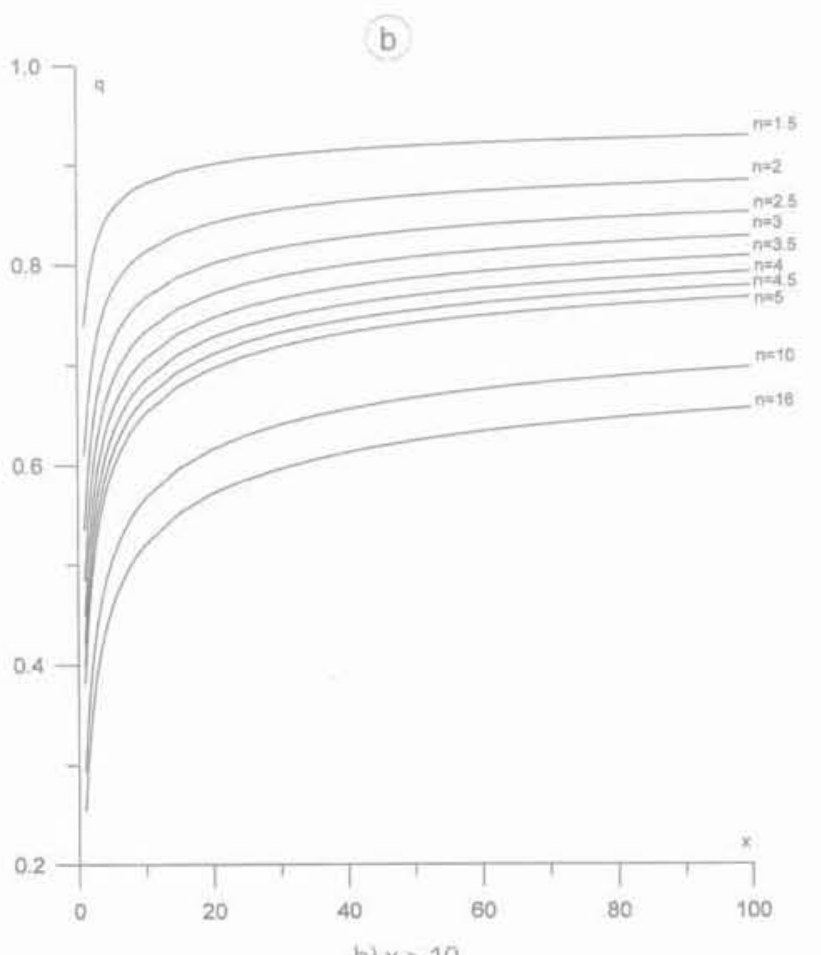

b) $x>10$

FG. 3 Détermination de $x=\lambda_{1} \sqrt{\alpha}$ pour des cavités allongées.

Evaluation of $x=\lambda \cdot \sqrt{\alpha}$ in case of lengthened cavities. 


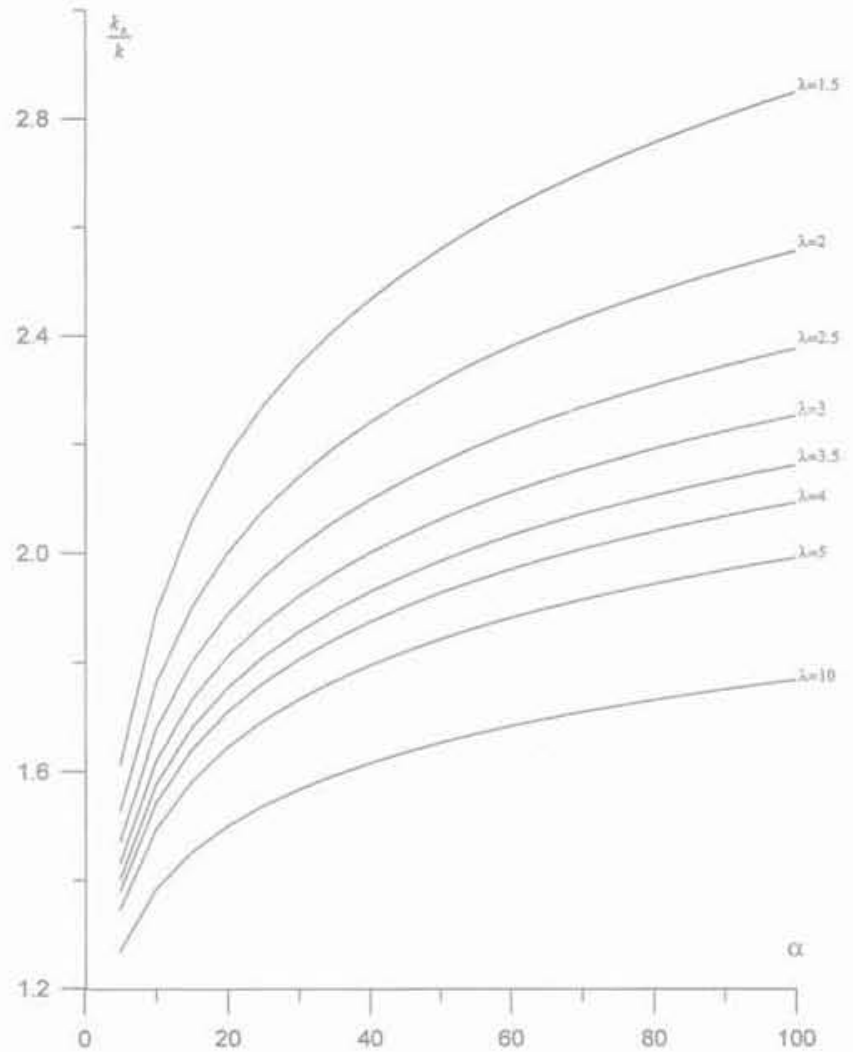

FG. 4 Évolution de $\frac{k_{\text {h }}}{k}$ en fonction de $\alpha$. Cavités allongées. $\mathrm{k}$ Variation of $\frac{k_{h}}{k}$ according to $\alpha$. Lengthened
cavities.

- d'autre part que, toutes choses égales par ailleurs, les écarts entre $k_{h}$ et $k$ évoluent beaucoup moins rapidement lorsque $\alpha$ augmente ;

- enfin que, dans le domaine des valeurs réalistes de $\alpha$ et des élancements usuels des cavités d'essai, la valeur de $\mathrm{k}$, excède rarement $3 \mathrm{k}$, ce qui peut poser quelques problèmes compte tenu du fait qu'il est difficile de déterminer un coefficient de perméabilité à moins d'une demi-puissance de 10 près. Mais en revanche, dans cette plage de variation de $\frac{k_{h}}{k}$, les coefficients d'anisotropie varient de 10 à 100. On voit donc tout l'intérêt que l'on a à augmenter le plus que l'on peut le contraste des élancements.

\section{3}

\section{Cavités de faible hauteur en milieu indéfini}

\section{$3.3,1$}

\section{Analyse du problème}

On appelle cavités de faible hauteur celles dont les élancements sont inférieurs à 1,5, et les expressions de leurs coefficients de forme ont été données dans le tableau précédent. Par rapport aux cavités allongées, le problème des cavités de faible hauteur se complique, car c'est pour de telles cavités que les coefficients de forme des domaines réel et transformé peuvent changer d'expression. Ce point va être examiner un peu plus en détail.
Supposons donc que l'on réalise le premier essai Lefranc dans une cavité réelle dont l'élancement soit tel que $0,7 \geq \lambda_{1}>0,3$, ce quî correspond à une demisphère.

Pour que la cavité du milieu transformé appartienne à la même famille géométrique, il faut que l'on ait :

$$
\frac{0,7}{\sqrt{\alpha}} \geq \lambda_{1}>0,3
$$

Si par exemple $\alpha=2$ (valeur minimale et quelque peu irréaliste) on obtient :

$$
0,5>\lambda_{1}>0,3
$$

Avec un diamètre courant de forage $B=0,12 \mathrm{~m}$, on est alors conduit à des cavités de longueurs comprises entre 6 et $3,6 \mathrm{~cm}$. On ne dispose donc que d'une marge de manceuvre de l'ordre de 2 centimètres seulement, ce quí n'est pas significatif et impossible à respecter dans la pratique, surtout dans des forages profonds. Cette marge diminue encore si le coefficient d'anisotropie est supérieur à 2, ce qui est le cas général. Pour $\alpha=4$, on $\mathrm{a}$, en effet, $0,35 \geq \lambda_{1}>0,3$, c'est-à-dire des longueurs de cavité comprises entre 5,2 et $4,5 \mathrm{~cm}$, soit une marge de $0,7 \mathrm{~cm}$, ce qui est ridicule.

On peut donc admettre que, pratiquement, à toute cavité réelle de type hémisphérique correspond, dans le milieu transformé, une cavité de type sphérique et pouvant même atteindre le type ellipsoïdal si $\alpha>5$. On aura donc en général :

$$
0,7 \geq \lambda_{1}>0,3 \Rightarrow 1,5 \geq \lambda_{1} \sqrt{\alpha}>0,7
$$

En ce qui concerne le deuxième essai Lefranc, il doit être réalisé avec une cavité d'élancement $\lambda$, significativement plus grand que $\lambda_{1}$ et, en tout état de cause, supérieur à 2 , ce qui conduit à $1,4 \geq \lambda_{2}>0,6$, c'est-à-dire pratiquement à une cavité de type sphérique. Dans le milieu transformé, la cavité correspondante sort alors de la famille sphérique pour entrer dans la famille ellipsoïdale dès que $\alpha=4$. Pour $\alpha \leq 4$, les seules valeurs possibles de $\lambda_{2}$ pour que $\lambda_{2} \sqrt{\alpha}$ reste dans la famille sphérique sont :

$$
\begin{array}{ll}
1,1 \geq 2>0,7 & \text { pour } \alpha=2 \\
0,87 \geq \lambda_{2}>0,7 & \text { pour } \alpha=3 \\
0,75 \geq \lambda_{2}>0,7 & \text { pour } \alpha=4
\end{array}
$$

On constate, ici aussi, que la marge de manceuvre, quoique plus importante que précédemment, reste encore faible et que dans le domaine transformé, on sera vite sorti de la famille géométrique du domaine réel. Mais on ne peut pas exclure totalement l'éventualité que les cavités des domaines réel et transformé restent dans la même famille.

En examinant les autres combinaisons, possibles et réalistes, entre les familles géométriques des deux domaines, on voit apparaître cinq cas qui sont rassemblés dans le tableau II ci-après.

Pour résoudre le problème, qui est la détermination de $\alpha$, il faudrait savoir dans quelle catégorie se situe la cavité du domaine transformé. Or, cette catégorie dépend précisément de $\alpha$ ! On se trouve donc dans une impasse.

On peut alors essayer d'effectuer le calcul pour les différentes catégories d'élancements transformés $\lambda_{i} \sqrt{\alpha}$ et comparer les valeurs de $\alpha$ ainsi obtenues. Si elles ne sont pas trop différentes, on pourra admettre qu'on a résolu le problème. Dans le cas contraire, on se contentera de donner une fourchette de valeurs. 
TABLEAUII Élancement des cavités de faible hauteur.

\begin{tabular}{l|l}
\hline Milieu réel & Milieu transformé \\
\hline \multirow{4}{*}{$0,7 \geq \lambda_{1}>0,3$} & $1,5 \geq \lambda_{1} \sqrt{\alpha}>0,7$ \\
& $1,5 \geq \lambda_{2} \sqrt{\alpha}>0,7$ \\
\cline { 2 - 2 } $1,5>\lambda_{2}>0,7$ & $1,5 \geq \lambda_{1} \sqrt{\alpha}>0,7$ \\
& $\lambda_{2} \sqrt{\alpha}>1,5$ \\
\cline { 2 - 2 } & $\lambda_{1} \sqrt{\alpha}>1,5$ \\
& $\lambda_{2} \sqrt{\alpha}>1,5$ \\
\hline \multirow{2}{*}{$1,5 \geq \lambda_{1}>0,7$} & $1,5 \geq \lambda_{1} \sqrt{\alpha}>0,7$ \\
$\lambda_{2}>1,5$ & $\lambda_{2} \sqrt{\alpha}>1,5$ \\
\cline { 2 - 2 } & $\lambda_{1} \sqrt{\alpha}>1,5$ \\
& $\lambda_{2} \sqrt{\alpha}>1,5$ \\
\hline
\end{tabular}

On voit donc que, ainsi qu'on l'a déjà signalé, le seul cas où il n'y a aucune ambiguité est celui des cavités réelles allongées. C'est pourquoi, puisque, d'une façon générale, on est libre de donner aux cavités expérimentales les hauteurs que l'on veut, il faudra toujours essayer de se ramener à des élancements supérieurs à 1,5.

Il en résulte que le cas des cavités de faible hauteur ne présente pas un grand intérêt pratique, sauf peutêtre celui de la cavité initiale de hauteur négligeable, c'est-à-dire $\lambda_{1}<0,3$, à condition d'adopter pour la cavité du deuxième essai un élancement $\lambda_{2}>1,5$.

Il est toutefois un autre cas, assez rare il faut bien le reconnaitre, où l'on peut avoir recours aux formules des cavités de faibles hauteurs, c'est celui où l'on connait, par des considérations géologiques ou statistiques, un ordre de grandeur de la valeur de $\alpha$ que l'on juge suffisamment représentatif et où l'on cherche à dissocier $k_{b}$ et $k$, ce qui ne peut se faire que si l'on dispose, soit d'une deuxième relation dans laquelle intervient l'un de ces deux coefficients de perméabilité, soit d'un moyen d'en évaluer directement l'un d'entre eux.

C'est pour ces deux raisons, mais également aussi dans un souci d'exhaustivité, qu'on a étudié le cas des cavités de faible hauteur malgré leur intérêt pratique assez limité.

\section{8 .9 .9}

Cavités réelles d'élancement $0,7>\lambda_{1}>0,3$ et $1,5>\lambda_{2}>0,7$

a) Cavités transformées d'élancements $1,5 \geq \lambda_{i} \sqrt{\alpha}>0,7$

En considérant les coefficients de forme du domaine réel et du domaine transformé, les relations (4) et (7) appliquées à chacun des deux essais Lefranc permettent d'écrire :

$$
\begin{aligned}
& Q_{1}=\pi \mathrm{k}_{1} \mathrm{~h}_{1} \mathrm{~B} \sqrt{\frac{4 \lambda_{1}+1}{2}}=\pi \mathrm{k}_{\mathrm{h}} \mathrm{h}_{1} \mathrm{~B} \sqrt{\frac{4 \lambda_{1} \sqrt{\alpha}+1}{\alpha}} \\
& \mathrm{Q}_{2}=\pi \mathrm{k}_{2} \mathrm{~h}_{2} \mathrm{~B} \sqrt{4 \lambda_{2}+1}=\pi \mathrm{k}_{\mathrm{h}} \mathrm{h}_{2} \mathrm{~B} \sqrt{\frac{4 \lambda_{2} \sqrt{\alpha}+1}{\alpha}}
\end{aligned}
$$

En posant: $\lambda_{2}=\mathrm{n} \lambda_{1} \quad \lambda_{1} \sqrt{\alpha}=x \quad q=\frac{h_{1} Q_{2}}{h_{2} Q_{1}}$ on déduit des deux relations précédentes:

$$
\begin{aligned}
& q=\sqrt{\frac{4 n x+1}{4 x+1}} \\
& \text { d'où : } \alpha=\left[\frac{q^{2}-1}{4 \lambda_{1}\left(q^{2}-n\right)}\right]^{2} \\
& \frac{k_{h}}{k_{1}}=\sqrt{\frac{\alpha\left(4 \lambda_{1}+1\right)}{8 \lambda_{1} \sqrt{\alpha}+2}} \text { et } \frac{k_{h}}{k_{2}}=\sqrt{\frac{\alpha\left(4 \lambda_{2}+1\right)}{4 \lambda_{2} \sqrt{\alpha}+1}} \\
& \mathrm{k}_{\mathrm{h}}=\frac{\mathrm{Q}_{\mathrm{i}}}{\pi \mathrm{h}_{\mathrm{i}} \mathrm{B}} \sqrt{\frac{\alpha}{4 \lambda_{i} \sqrt{\alpha}+1}} \\
& \text { ( } \mathrm{i}=1 \text { et } 2 \text { ) }
\end{aligned}
$$

Les valeurs de $k_{1}$ et $k_{2}$ sont celles obtenues à partír des deux essais Lefranc normalisés.

b) Cavités transformées d'élancement $1,5 \geq \lambda, \sqrt{\alpha}>0,7$ et $\lambda_{2} \sqrt{\alpha}>1,5$

En raisonnant comme précédemment, on obtient tous calculs faits ;

$$
q=\frac{x}{\sqrt{4 x+1 \cdot \operatorname{Argsh}(n x)}}
$$

avec: $\mathrm{q}=\frac{\mathrm{h}_{1} \mathrm{Q}_{2}}{2 \mathrm{n} \mathrm{h}_{2} \mathrm{Q}_{1}}$

L'équation précédente se résout numériquement ou à l'aide de la table numérique 3 de l'annexe 2 ou des courbes de la figure 5 .

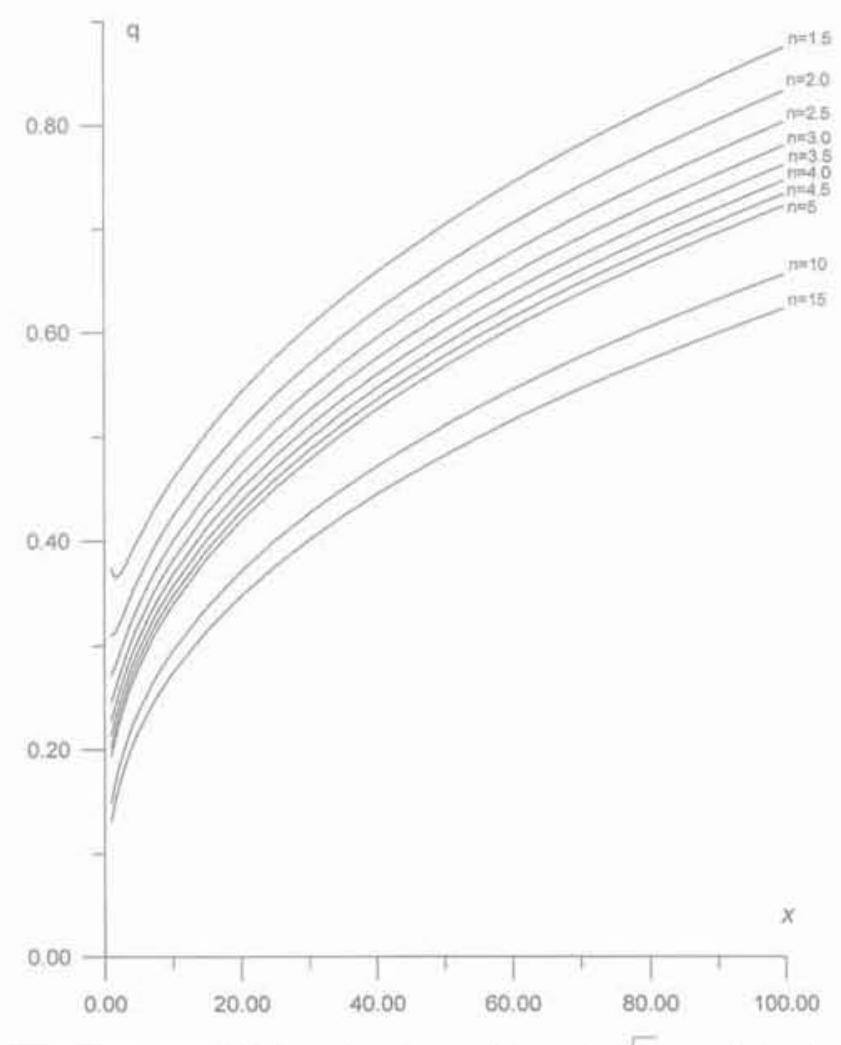

FG. 5 Détermination de $x=\lambda_{1} \sqrt{\alpha}$ cavités de faible hauteur.

Evaluation of $x=\lambda_{1} \sqrt{\alpha}$ in case of short heigth cavities. 
Connaissant $\alpha=\left(\frac{x}{\lambda_{1}}\right)^{2}$, on peut alors évaluer $k_{h}$ à partir des relations suivantes:

$$
\frac{k_{h}}{k_{1}}=\sqrt{\frac{\alpha\left(4 \lambda_{1}+1\right)}{8 \lambda_{1} \sqrt{\alpha+2}}} \text { et } \frac{k_{h}}{k_{2}}=\frac{\sqrt{4 \lambda_{2}+1}}{2 \lambda_{2}} \cdot \operatorname{Argsh}\left(\lambda_{2} \sqrt{\alpha}\right)
$$

ou :

$$
\mathrm{k}_{\mathrm{h}}=\frac{\mathrm{Q}_{1}}{\pi \mathrm{h}_{1} \mathrm{~B}} \sqrt{\frac{\alpha}{4 \lambda_{1} \sqrt{\alpha}+1}}=\frac{\mathrm{Q}_{2}}{2 \pi \lambda_{2} \mathrm{~h}_{2} \mathrm{~B}} \operatorname{Argsh}\left(\lambda_{2} \sqrt{\alpha}\right)
$$

c) Cavités transformées d'élancements $\lambda_{1} \sqrt{\alpha}>1,5$

En ce qui concerne la détermination de $\alpha$, on retrouve l'équation (12) des cavités allongées puisque les cavités du domaine transformé appartiennent à cette famille:

$$
\mathrm{q}=\frac{\operatorname{Argsh} \mathrm{x}}{\operatorname{Argsh} \mathrm{n} x}
$$

avec: $\quad \mathrm{q}=\frac{\mathrm{h}_{1} \mathrm{Q}_{2}}{\mathrm{nh}_{2} \mathrm{Q}_{1}}$

on a par ailleurs :

$$
\begin{aligned}
\frac{k_{h}}{k_{1}} & =\frac{\sqrt{2 \lambda_{1}+0,5}}{2 \lambda_{1}} \cdot \operatorname{Argsh}\left(\lambda_{1} \sqrt{\alpha}\right) \\
\frac{k_{h}}{k_{2}} & =\sqrt{\frac{4 \lambda_{2}+1}{2 \lambda_{2}}} \cdot \operatorname{Argsh}\left(\lambda_{2} \sqrt{\alpha}\right) \\
k_{h} & =\frac{Q_{i}}{2 \pi \lambda_{i} h_{i} B} \cdot \operatorname{Argsh}\left(\lambda_{i} \sqrt{\alpha}\right)
\end{aligned}
$$

avec $: \mathrm{i}=1$ et 2 .

\section{3 .3}

\section{Cavités réelles d'élancement $1,5 \geq \lambda_{1}>0,7$ et $\lambda_{9}>1,5$}

a) Cavités transformées d'élancements $1,5>\lambda_{1} \sqrt{\alpha}>0,7$ et $\lambda_{2} \sqrt{\alpha}>1,5$

Dans le domaine transformé, les cavités appartiennent aux mêmes familles qéométriques que dans le cas du $\S 3.3 .2 . b$. Pour déterminer $\alpha$ on retrouve donc l'équation (15):

$$
q=\frac{x}{\sqrt{4 x+1 \cdot} \cdot \operatorname{Argsh}(n x)}
$$

avec: $\mathrm{q}=\frac{\mathrm{h}_{1} \mathrm{Q}_{2}}{2 \mathrm{nh}_{2} \mathrm{Q}_{1}}$

Mais on a en revanche:

$$
\frac{\mathrm{k}_{\mathrm{h}}}{\mathrm{k}_{1}}=\sqrt{\frac{\alpha\left(4 \lambda_{1}+1\right)}{4 \lambda_{1} \sqrt{\alpha}+1}} \text { et } \frac{\mathrm{k}_{\mathrm{h}}}{\mathrm{k}_{2}}=\frac{\operatorname{Argsh}\left(\lambda_{2} \sqrt{\alpha}\right)}{\operatorname{Argsh} \lambda_{2}}
$$

Puis on retrouve, ce qui est normal :

$$
\mathrm{k}_{\mathrm{h}}=\frac{\mathrm{Q}_{1}}{\pi \mathrm{h}_{1} \mathrm{~B}} \sqrt{\frac{\alpha}{4 \lambda_{1} \sqrt{\alpha}+1}}=\frac{\mathrm{Q}_{2}}{2 \pi \lambda_{2} \mathrm{~h}_{2} \mathrm{~B}} \operatorname{Argsh}\left(\lambda_{2} \sqrt{\alpha}\right)
$$

b) Cavités transformées d'élancement $\lambda_{i} \sqrt{\alpha}>1,5$

Pour ce qui est de la détermination de $\alpha$, on retrouve l'équation (12) comme en 3.2 puisqu'on est dans la même configuration quant à l'espace transformé.
En revanche, on a :

$\frac{k_{h}}{k_{1}}=\frac{\sqrt{4 \lambda_{1}+1}}{2 \lambda_{1}} \cdot \operatorname{Argsh}\left(\lambda_{1} \sqrt{\alpha}\right)$ et $\frac{k_{h}}{k_{2}}=\frac{\operatorname{Argsh}\left(\lambda_{2} \sqrt{\alpha}\right)}{\operatorname{Argsh} \lambda_{2}}$

Mais on a toujours :

$$
\mathrm{k}_{\mathrm{h}}=\frac{\mathrm{Q}_{\mathrm{i}}}{2 \pi \lambda_{1} \mathrm{~h}_{\mathrm{i}} \mathrm{B}} \operatorname{Argsh}\left(\lambda_{i} \sqrt{\alpha}\right)
$$

L'ensemble des résultats obtenus ci-dessus pour les cavités de faible hauteur est récapitulé dans le tableau III du paragraphe 5 .

\section{6}

\section{Cavité initiale d'élancement nul}

La cavité d'élancement nul n'est autre que le disque limitant le fond du forage.

On sait que, dans ce cas, on a :

$$
\mathrm{Q}=2 \mathrm{kh} \mathrm{B}
$$

En opérant la même transformation affine que dans les cas précédents, on obtient:

$$
\mathrm{Q}=2 \mathrm{k}_{\mathrm{h}} \mathrm{h} \frac{\mathrm{B}}{\sqrt{\alpha}}
$$

d'oủ l'on tire :

$$
k_{h}=k \sqrt{\alpha}
$$

c'est-à-dire :

$$
\mathrm{k}=\sqrt{\mathrm{k}_{\mathrm{h}} \mathrm{k}_{\mathrm{v}}}
$$

On voit donc que, contrairement à une idée très répandue, l'essai par le disque qui limite le fond du forage ne donne pas le coefficient de perméabilité verticale mais un coefficient qui est égal à la moyenne géométrique des coefficients de perméabilité verticale et horizontale.

Soient toujours :

$h_{1}$ : la variation du niveau de l'eau sous le débit $Q_{1}$ dans la cavité de hauteur nulle :

$h_{2}$ : la variation du niveau de l'eau sous le débit $Q_{2}$ dans lạ cavité d'élancement $\lambda$.

On a alors dans tous les cas :

$$
\begin{aligned}
& Q_{1}=\frac{2}{\sqrt{\alpha}} k_{h} h_{1} B \\
& Q_{2}=\frac{m(\lambda \sqrt{\alpha})}{\sqrt{\alpha}} k_{h} h_{2} B
\end{aligned}
$$

d'où :

$$
\frac{2 h_{1} Q_{2}}{h_{2} Q_{1}}=m(\lambda \sqrt{\alpha})
$$

Si on pose toujours $\mathrm{x}=\lambda \sqrt{\alpha}$, la relation précédente se ramène à une équation de type $q=f(x)$ qui s'explicite immédiatement à partir des expressions analytiques des coefficients de forme donnés précédemment (§2).

Mais il faut, ici aussi, estimer à quelle famille géométrique appartient la cavité du domaine transformé, ce qui amène à retenir quatre cas :

- $0,7 \geq \lambda>0,3 \quad$ avec $\quad 1,5 \geq \lambda \sqrt{\alpha}>0,7$ 
- $1,5 \geq \lambda>0,7 \quad$ avec $\quad 1,5 \geq \lambda \sqrt{\alpha}>0,7$

- $1,5>\lambda>0,7$

$$
\lambda \sqrt{\alpha} \geq 1,5
$$

$\cdot \lambda \geq 1,5$

$$
\lambda \sqrt{\alpha} \geq 1,5
$$

La formule (19) montre qu'en ce qui concerne I'équation $q=f(x)$ qui permet de déterminer le coefficient d'anisotropie, il n'y a, en fait, que deux cas à considérer, puisqu'il n'y a que deux familles géométriques dans le milieu transformé (sphère et ellipsoïde allongé), donc deux coefficients de forme et par conséquent deux équations en $x$. Ces deux cas suffisent également si l'on veut évaluer directement le coefficient de perméabilité horizontale. En revanche, si on veut déterminer $\mathrm{k}$ à partir des coefficients de perméabilité globale déduits des essais Lefranc selon les procédures de la norme AFP 94-132, il faut bien distinguer les quatre cas.

Les calculs se simplifient par rapport aux cas examinés dans les paragraphes précédents, mais se conduisent suivant les mêmes méthodes et ne posent aucune difficulté.

C'est ainsi que l'on obtient en particulier pour le cas où $\lambda \sqrt{\alpha} \geq 1,5$, auquel il faudra toujours essayer de se ramener:

$$
q=\frac{x}{\operatorname{Argsh} x}
$$

La résolution de cette équation nécessite un traitement numérique qui peut être remplacé par une approche approximative à l'aide de la courbe de la figure 6 ou de la table numérique 4 donnée dans l'annexe 2.

L'ensemble des résultats relatif à une cavité initiale de hauteur nulle est récapitulé dans le tableau IV du paragraphe 5.

$$
q=\frac{x}{\operatorname{Argsh} x}
$$

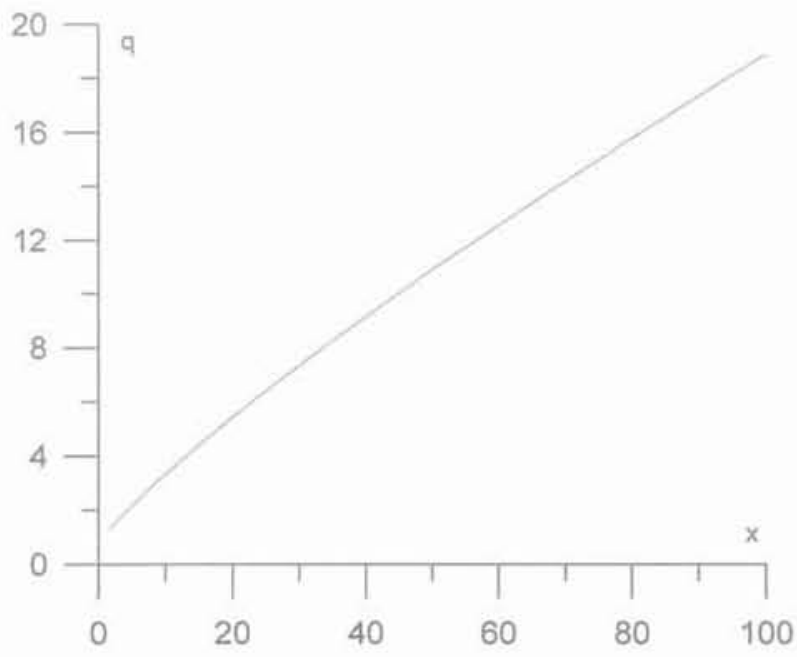

FG. 6

Détermination de $x=\lambda_{1} \sqrt{\alpha} \geq 1,5$.

Evaluation of $x=\lambda_{1} \sqrt{\alpha} \geq 1,5$.

\section{Cavité proche de l'une des limites de la nappe}

On n'examinera que les cas les plus courants représentés par les figures 7 et 8 .

On démontre $[2,5]$ que le coefficient de forme est donné par la relation :

$$
\frac{1}{\mathrm{~m}}=\frac{1}{\mathrm{~m}_{0}} \pm \frac{\mathrm{B}}{8 \pi \mathrm{D}}
$$

oú $\mathrm{m}_{\text {i }}$ est la valeur du coefficient de forme en milieu indéfini donné.

Le signe + correspond aux cas des figures 7 a et 8 , et le signe - à la figure $7 \mathrm{~b}$.

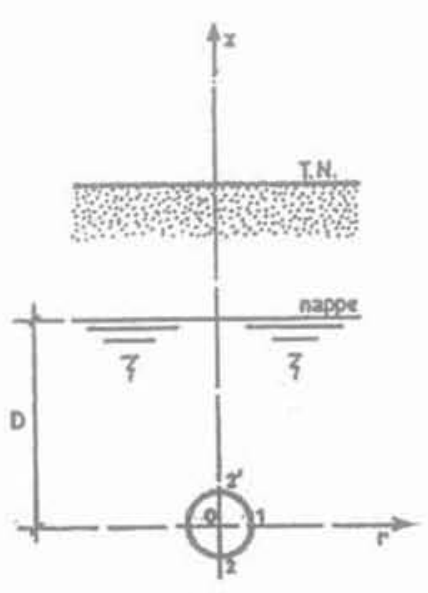

a

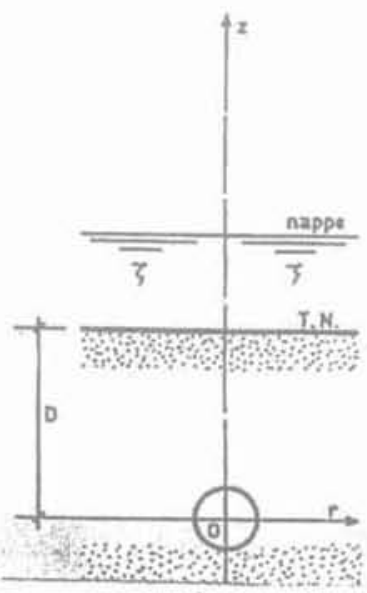

b
FG.7 Cavité à proximité de la limite supérieure de la nappe.

Cavity in the neighbourhood of the upper limit water level.

Pour une cavité allongée $(\lambda>1,5)$, la transformation affine précédente conduit, tous calculs faits, à la relation :

$$
Q=\frac{8 \pi \lambda}{4 \operatorname{Argsh}(\lambda \sqrt{\alpha}) \pm \delta} k_{h} h B
$$

avec $\delta=\frac{L}{D}$.

Si on effectue deux pompages sous des débits $Q_{1}$ et $\mathrm{Q}_{2}$, dans des cavités de longueur $\mathrm{L}_{1}$ et $\mathrm{L}_{2}$, le diamètre restant inchangé, on obtient :

$$
q=\frac{h_{1} Q_{2}}{n h_{2} Q_{1}}=\frac{4 \operatorname{Argsh}\left(\lambda_{1} \sqrt{\alpha}\right) \pm \delta_{1}}{4 \operatorname{Argsh}\left(\lambda_{2} \sqrt{\alpha}\right) \pm \delta_{2}}
$$

avec toujours: $\mathrm{n}=\frac{\lambda_{2}}{\lambda_{1}}$ et $\mathrm{x}=\lambda_{1} \sqrt{\alpha}$.

La relation précédente peut alors s'écrire :

$$
q=\frac{4 \operatorname{Argsh} x \pm \delta_{1}}{4 \operatorname{Argsh}(n x) \pm n \delta_{1}}
$$

Cette équation se résout facilement par méthode numérique. On voit que lorsque $D \rightarrow \infty, \delta \rightarrow 0$. On retrouve alors l'équation (12). On laisse au lecteur le soin d'établir les relations correspondant aux autres valeurs de $\lambda$, ce qui ne présente aucune difficulté. 

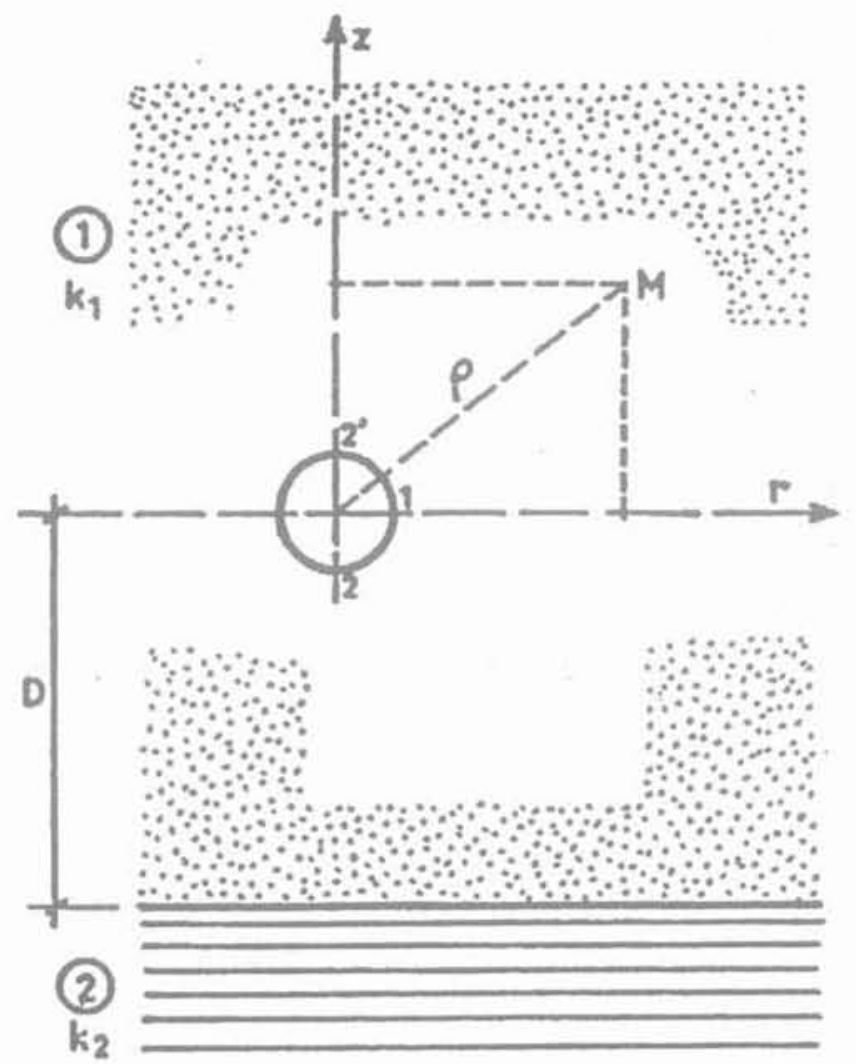

FG. 8 . Cavité à proximité du substratum étanche supposé infiniment profond.

Cavity in the neighbourhood of the infinitely deep supposed waterproff bedrock.

\section{5}

\section{Application pratique}

Dans une formation alluvionnaire constituée de graves sableuses, on a réalisé un puits en gros diamètre par battage d'un tube de $0,50 \mathrm{~m}$ de diamètre extérieur descendu à 30 mètres de profondeur, c'est-à-dire à 24 mètres sous le niveau statique de la nappe.

Une fois le forage terminé, on a descendu à l'intérieur du tube de forage, un tube crépiné de $350 \mathrm{~mm}$ de diamètre et mis en place, dans l'espace annulaire un filtre en gravillon calibré. Le tube de forage a été ensuite remonté d'une hauteur de $2,50 \mathrm{~m}$ qui constitue donc la hauteur de la cavité de filtration.

Après développement, on a procédé à un premier pompage sous un débit de $85 \mathrm{~m}^{3} / \mathrm{h}$. Une fois ce pompage terminé, le tube de forage a été remonté d'une hauteur encore égale à 2,50 m. La cavité de filtration avait alors une hauteur de 5 mètres. Un deuxième pompage a été ensuite réalisé sous un débit de $180 \mathrm{~m}^{3} / \mathrm{h}$.

Dans les deux cas, on a considéré que le diamètre de la cavité était de $0,50 \mathrm{~m}$ et on a calculé le coefficient de perméabilité apparent k à l'aide de la formule (4).
Les données du problème et les valeurs de $\mathrm{k}$ sont donc les suivantes:

\begin{tabular}{c|r|r|r|r|r|r|r}
\hline & $Q\left(\mathrm{~m}^{3} / \mathrm{h}\right)$ & $\mathrm{h}(\mathrm{m})$ & $\mathrm{B}(\mathrm{m})$ & $\mathrm{I}(\mathrm{m})$ & $\lambda$ & $\mathrm{m}$ & $\mathrm{K}(\mathrm{m} / \mathrm{s})$ \\
\hline Pompage 1 & 85 & 1,83 & 0,5 & 2,5 & 5 & 13,6 & $1,91.10^{-3}$ \\
\hline Pompage 2 & 180 & 2,31 & 0,5 & 5,0 & 10 & 21,0 & $2,1.10^{-3}$ \\
\hline
\end{tabular}

\section{Calcul de $\alpha$}

S'agissant d'une cavité allongée $(\lambda>1,5)$, on utilise la formule (12), avec d'après le tableau ci-dessus :

$$
\begin{aligned}
& n=\frac{10}{5}=2 \\
& \mathrm{q}=\frac{\mathrm{h}_{1} \mathrm{Q}_{2}}{2 \mathrm{~h}_{2} \mathrm{Q}_{1}}=\frac{1,83 \times 180}{2 \times 2,3 \times 85}=0,8388 \# 0,839
\end{aligned}
$$

En portant cette valeur sur la courbe $n=2$ de la figure 3 , ou en utilisant la table numérique de l'annexe $\mathrm{A} 2$, on voit que pour $\mathrm{q}=0,839$, on obtient :

$$
\mathrm{x}=18,6
$$

d'où :

$$
\alpha=\left(\frac{x}{\lambda_{1}}\right)^{2}=\left(\frac{18,6}{5}\right)^{2}=13,8 \# 14
$$

Calcul de $k_{h}$ et $k_{v}$

On utilisera la formule (13) ou les courbes de la figure 5 correspondant à $\lambda_{1}=5$ pour le premier pompage et $\lambda_{2}=10$ pour le deuxième pompage avec $\alpha=14$.

On obtient alors : - pompage $1: \frac{k_{h}}{k}=1,57$ d'où $k_{h}=1,57 \times 1,91.10^{-3}=$
$3,10^{-3} \mathrm{~m} / \mathrm{s}$;

- pompage 2: $\frac{\mathrm{k}_{\mathrm{h}}}{\mathrm{k}}=1,44$ d'où $\mathrm{k}_{\mathrm{h}}=1,44 \times 2,1.10^{-3}=$ $3,02 \cdot 10^{-3} \# 3 \cdot 10^{-3} \mathrm{~m} / \mathrm{s}$.

On a done :

$$
\begin{aligned}
& \mathrm{k}_{\mathrm{h}}=3 \cdot 10^{-3} \mathrm{~m} / \mathrm{s} \\
& \mathrm{k}_{\mathrm{v}}=\frac{3}{14} \cdot 10^{-3}=2 \cdot 1 \cdot 10^{-4} \mathrm{~m} / \mathrm{s} \text { arrondi à } 2 \cdot 10^{-4} \mathrm{~m} / \mathrm{s}
\end{aligned}
$$

on retiendra finalement :

$$
\alpha=\frac{k_{\mathrm{h}}}{\mathrm{k}_{\mathrm{v}}}=15
$$

On remarquera que les valeurs de $k_{1}$ et $k_{2}$ correspondant aux deux pompages sont très voisines bien que les débits soient très différents.

On voit donc que, dans le domaine pratique, les résultats obtenus seront d'autant plus fiables que les paramètres de calculs seront contrastés, en particulier les coefficients de forme et les charges hydrauliques dans la cavité.

\section{Recommandations pratiques concernant les dispositions expérimentales}

Théoriquement, la méthodologie expérimentale est simple puisqu'il ne s'agit que de faire varier la longueur 
des cavités de filtration. Mais c'est la mise en pratique de cette méthodologie qui peut poser problème en fonction de la nature des matériaux à étudier. Deux cas sont à considérer, selon que le sol rencontré est doué ou non de cohésion.

a) Si la cohésion du sol est suffisante pour assurer la stabilité des parois du forage, on procédera selon le principe de la figure 9 .

Lorsque l'isolation hydraulique de la cavité est assurée par un obturateur gonflable, on réalisera la cavité initiale de hauteur $L_{3}$ à la base de la zone à tester (Fig.9a).

Après la réalisation du premier essai, on sortira l'obturateur du forage et on ajoutera du matériau filtrant pour augmenter, vers le haut, la longueur de la cavité jusqu'à la valeur $L_{2}$ (Fig. 9b), et on réalisera le deuxième essai. On pourra ensuite répéter le processus si on veut un troisième élancement de cavité.

Lorsque l'isolation hydraulique de la cavité est assurée par un bouchon d'argile, il faut, pour modifier la longueur de la cavité, détruire ce bouchon. Or, si on développe la cavité vers le haut, la nouvelle cavité recouvrira l'emplacement du premier bouchon, et, malgré toutes les précautions qui peuvent être prises, on ne pourra jamais être certain qu'aucune particule argileuse ne soit venue polluer le filtre.

C'est pourquoi, puisque, de toute façon il faut détruire le premier bouchon d'argile, on estime qu'il est préférable d'allonger la cavité par le bas de façon à ce que les différentes poches filtrantes se trouvent toujours au-dessous de la zone des bouchons d'argile précédents. C'est la configuration de la figure 9c. Dans ce cas, la cavité initiale devra être réalisée dans la partie haute de la zone à tester.

b) Si la cohésion du sol ne permet pas d'assurer l'équilibre des parois du forage, celui-ci devra être obligatoirement tubé et c'est le tube de forage, avec sabot non débordant, qui assurera l'étanchéité des parois et l'isolation hydraulique de la cavité de filtration.

La cavité initiale devra être réalisée à la base de la zone à tester et les allongements successifs se feront vers le haut par remontées successives du tube de forage conformément au schéma de la figure 10.

S'il n'est pas possible d'atteindre avec un seul tubage les zones à tester parce qu'elles sont trop profondes, on pourra télescoper le forage, mais il sera nécessaire de réaliser un bouchon d'argile entre les deux tubes et d'effectuer les allongements de cavité par le haut en remontant le tube intérieur, le tube extérieur restant fixe (Fig. 11).

c) Une autre méthode, valable quelle que soit la nature du sol étudié, consiste à réaliser deux essais Lefranc, avec des cavités d'élancements différents, dans deux forages voisins, les centres des cavités se trouvant à la même profondeur. Pour éviter toute interférence entre les deux forages, ceux-ci devront être distants d'environ quinze diamètres.

Cette méthode, techniquement très intéressante est évidemment plus onéreuse car elle oblige à doubler les forages.

Pour chaque phase d'expérimentation, et quelle que soit la méthode adoptée pour la réalisation des cavités, l'exécution des essais devra suivre rigoureusement les prescriptions de la norme NFP 94-132. (a)

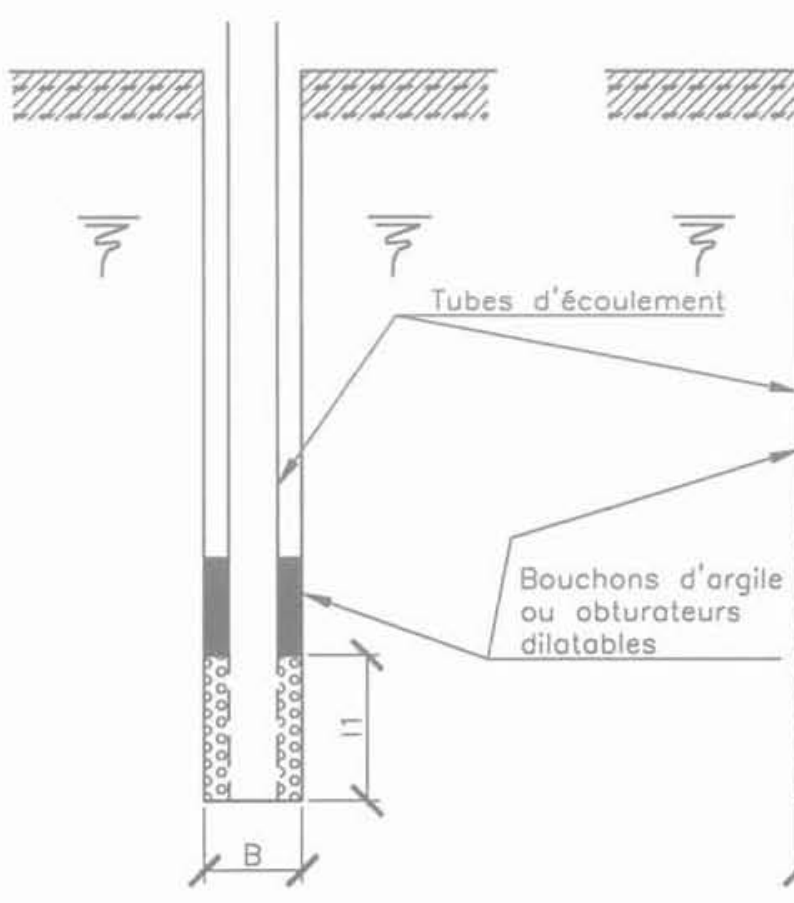

(b)

(C)

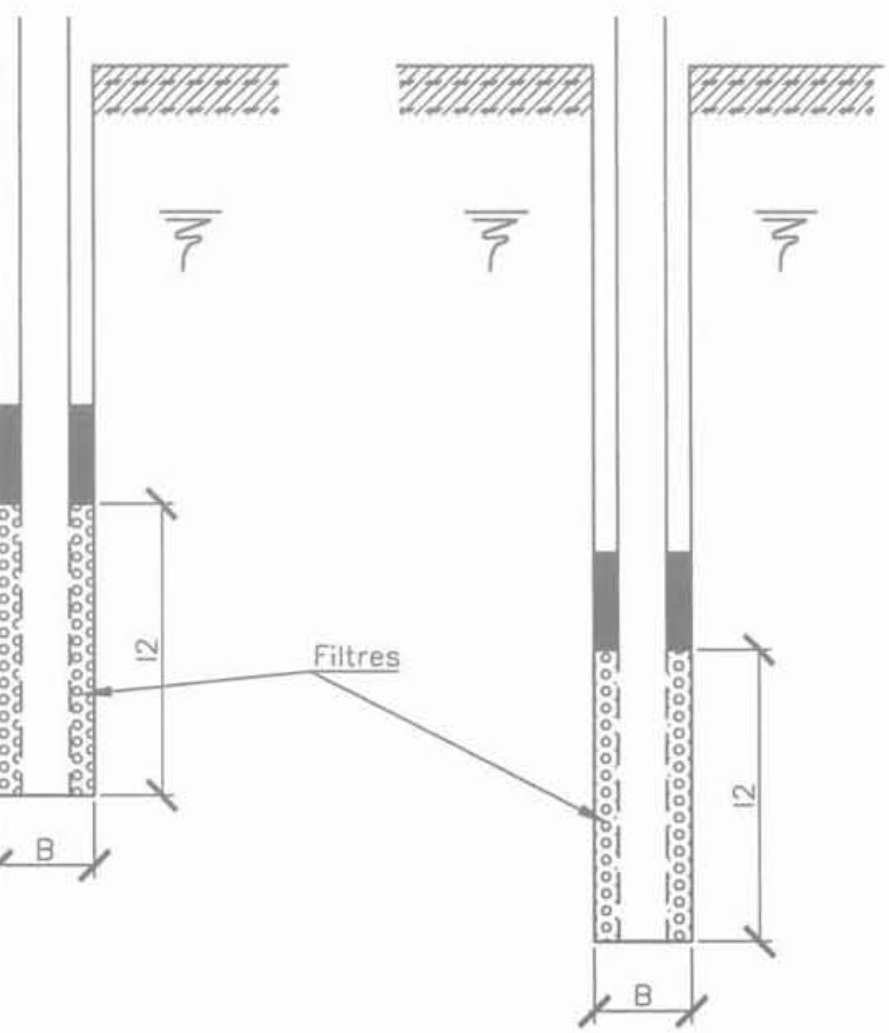

FG.9 Réalisation des cavités en terrain cohérent. Carrying out of cavities in cohesive soils. 


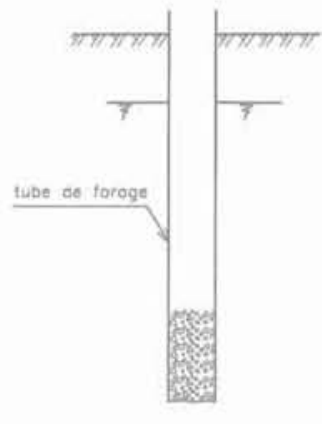

(1)

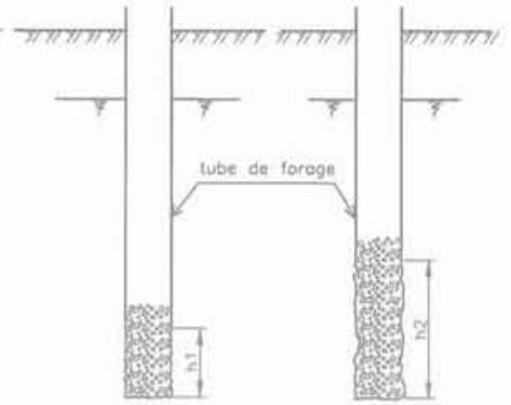

(2)
(3)
Fị. 10 Réalisation des cavités dans les sols sans cohésion.

Carrying out of cavities in cohesionless soils.

\section{Récapitulation des procédures d'interprétation}

On a récapitulé dans les tableaux III et IV ci-après les procédures d'interprétation développées dans la présente étude et qui sont basées sur la résolution, en général par méthode numérique, de l'équation $q=f(x)$.

Dans ces tableaux, on a fait figurer systématiquement les expressions des rapports $\frac{k_{h}}{k}$ oủ $k$ est la valeur du coefficient de perméabilité globale déterminé selon la norme NFP 94-132.

Dans certains cas, en effet, pour évaluer $k_{h}$, il est plus rapide d'utiliser cette relation que de proceder au calcul direct, puisque de toute façon le calcul de $k$ doit être effectué.

\section{6}

\section{Conclusion}

La notion d'anisotropie des sols est déjà ancienne, mais, bien que les méthodes de calcul numériques actuelles puissent permettre d'en tenir compte facilement dans les projets, elle n'a été que rarement utilisée dans la pratique courante, à cause des difficultés que présente la détermination du paramètre qui la caractérise et qui est défini comme le rapport des coefficients de perméabilité horizontale et verticale.

Jusqu'à présent, ce coefficient d'anisotropie a été évalué essentiellement en laboratoire, c'est-à-dire de façon très ponctuelle et dans les cas assez particuliers de sols permettant les prélèvements d'échantillons intacts. Or, tous les géotechniciens connaissent bien les difficultés de ces prélèvements et savent ce qui l'en est de la représentativité de tels échantillons.

Néanmoins, on pourrait penser que, s'agissant du rapport de deux coefficients de perméabilité mesurés séparément, l'erreur que l'on pourrait faire sur ce rapport est peut-être moins importante que celle que l'on peut faire sur l'évaluation de chacun de ces coefficients. Rien n'est moins sûr et il demeure que le champ d'application de la mesure en laboratoire reste très limité. C'est pourquoi, on a cherché à voir s'il était pos-

(1)

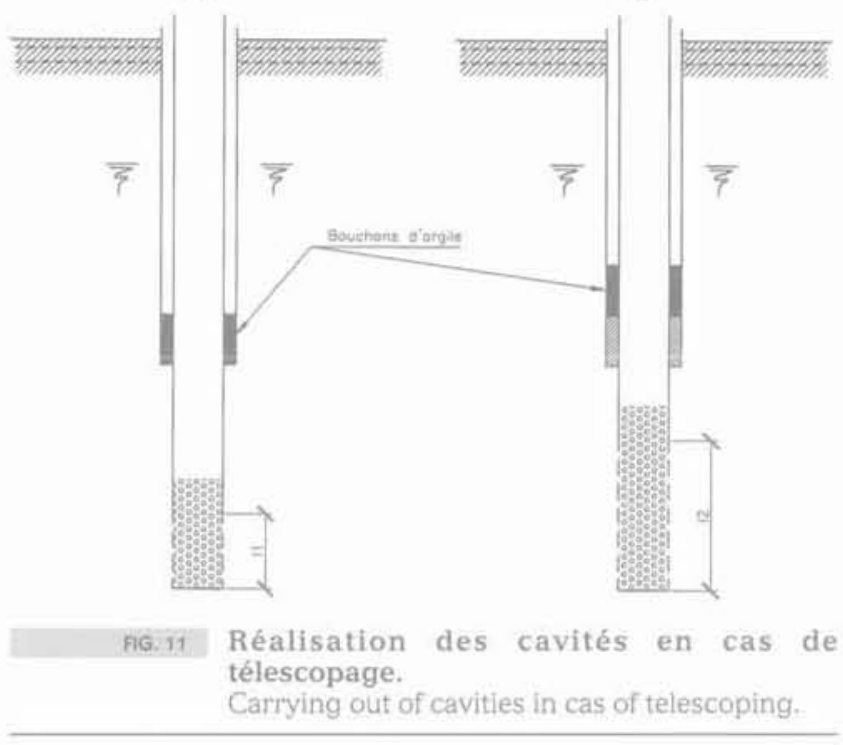

sible d'appréhender globalement ce coefficient d'anisotropie à l'aide des essais Lefranc classiques, qui sont actuellement très répandus et qui ont fait l'objet d'une normalisation.

Certes, l'étude présentée ici a un caractère très théorique, mais sous cet aspect, elle montre que la chose est tout à fait possible et que cette méthode, qui n'est d'ailleurs qu'une extension et une généralisation de celle avancée dès 1954 par G. Schneebeli, devrait conduire à des résultats plus fiables que ceux obtenus en laboratoire.

Toutefois, sur le plan de la mise en cuvre pratique, elle reste assez délicate et se heurte, comme tous les essais d'eau à la difficulté que l'on a d'obtenir des résultats significativement contrastés. Tous les spécialistes de l'hydraulique souterraine savent, en effet, l'importance de la plage d'incertitude qui entoure l'évaluation d'un coefficient de perméabilité et cette marge se répercute également sur l'évaluation du coefficient d'anisotropie.

C'est pourquoi, il ne faudra pas considérer les résultats obtenus dans toute leur rigueur arithmétique, mais plutôt ne voir en eux que des indicateurs de tendance. La méthode précédemment exposée n'a donc que la simple prétention de conduire, non pas à une valeur précise du coefficient d'anisotropie, mais à une fourchette de valeurs possibles et vraisemblables. Les méthodes numériques actuelles permettront alors d'effectuer plusieurs calculs dans cette fourchette et d'apprécier ainsi le poids de ce paramètre. Ce sera déjà un résultat digne d'intérêt pour toutes les études où intervient la circulation des eaux souterraines et notamment dans les problèmes de plus en plus fréquents et complexes que pose, à l'heure actuelle, la protection de l'environnement contre les pollutions de nappe par les rejets industriels les plus divers. 
TABLEAUIII Essais Lefranc en milieu indéfini - Cavités d'élancements non nuls.

\begin{tabular}{|c|c|c|c|c|c|c|}
\hline Milieu réel & $\begin{array}{l}\text { Milieu } \\
\text { transformé }\end{array}$ & q & $f(x)$ & $\alpha$ & $\frac{k_{i}}{k_{i}}$ & $k_{\mathrm{n}}$ \\
\hline $\begin{array}{l}\lambda_{1} \geq 1,5 \\
\lambda_{2} \geq 1,5\end{array}$ & $\begin{array}{l}\lambda_{1} \sqrt{\alpha} \geq 1,5 \\
\lambda_{2} \sqrt{\alpha} \geq 1,5\end{array}$ & $\frac{h_{1} Q_{2}}{\mathrm{nh}_{2} \mathrm{Q}_{1}}$ & $\frac{\operatorname{Argsh} x}{\operatorname{Argsh}(n x)}$ & $\left(\frac{x}{\lambda_{1}}\right)^{2}$ & $\frac{\operatorname{Argsh}\left(\lambda_{i} \sqrt{\alpha}\right)}{\operatorname{Argsh} \lambda_{i}}$ & $\frac{Q_{i}}{2 \pi \lambda_{i} h_{i} B} \operatorname{Argsh}\left(\lambda_{i} \sqrt{\alpha}\right)$ \\
\hline \multirow{3}{*}{$\begin{array}{l}0,7 \geq \lambda_{7}>0,3 \\
1,5 \geq \lambda_{2}>0,7\end{array}$} & $\begin{array}{l}1,5 \geq \lambda_{1} \sqrt{\alpha}>0,7 \\
1,5 \geq \lambda_{2} \sqrt{\alpha}>0,7\end{array}$ & $\frac{h_{1} Q_{2}}{h_{2} Q_{1}}$ & $\sqrt{\frac{4 n x+1}{4 x+1}}$ & {$\left[\frac{q^{2}-1}{4 \lambda_{1}\left(q^{2}-n\right)}\right.$} & $\begin{array}{l}\frac{k_{h}}{k_{1}}=\sqrt{\frac{\alpha\left(4 \lambda_{1}+1\right)}{8 \lambda_{1} \sqrt{\alpha}+1}} \\
\frac{k_{h}}{k_{2}}=\sqrt{\frac{\alpha\left(4 \lambda_{2}+1\right)}{4 \lambda_{2} \sqrt{\alpha+1}}}\end{array}$ & $\frac{Q_{1}}{\pi h_{1} B} \sqrt{\frac{\alpha}{4 \lambda_{1} \sqrt{\alpha}+1}}$ \\
\hline & $\begin{array}{l}1,5 \geq \lambda_{1} \sqrt{\alpha}>0,7 \\
\lambda_{2} \sqrt{\alpha}>1,5\end{array}$ & $\frac{h_{1} Q_{2}}{2 n h_{2} Q_{1}}$ & $\frac{x}{\sqrt{4 x+1, \operatorname{Argsh}(n x)}}$ & \multirow{4}{*}{$\left(\frac{x}{\lambda_{1}}\right)^{2}$} & $\begin{array}{l}\frac{k_{h}}{k_{1}}=\sqrt{\frac{a\left(4 \lambda_{1}+1\right)}{8 \lambda_{1} \sqrt{\alpha}+2}} \\
\frac{k_{h}}{k_{2}}=\frac{\sqrt{4 \lambda_{2}+1}}{2 \lambda_{2}} \cdot \operatorname{Argsh}\left(\lambda_{2} \sqrt{\alpha}\right)\end{array}$ & $\begin{array}{l}\frac{Q_{1}}{\pi h_{1} B} \sqrt{\frac{\alpha}{4 \lambda_{1} \sqrt{\alpha+1}}}= \\
\frac{Q_{z}}{2 \pi \lambda_{2} h_{2} B} \operatorname{Argsh}\left(\lambda_{2} \sqrt{\alpha}\right)\end{array}$ \\
\hline & $\begin{array}{l}\lambda_{1} \sqrt{\alpha}>1,5 \\
\lambda_{2} \sqrt{\alpha}>1,5\end{array}$ & $\frac{h_{1} Q_{2}}{\mathrm{nh}_{2} \mathrm{Q}_{1}}$ & $\frac{\operatorname{Argsh} x}{\operatorname{Argsh}(n x)}$ & & $\begin{array}{l}\frac{k_{n}}{k_{1}}=\frac{\sqrt{2 \lambda_{1}+0,5}}{2 \lambda_{1}} \cdot \operatorname{Argsh}\left(\lambda_{1} \sqrt{\alpha}\right) \\
\frac{k_{n}}{k_{2}}=\frac{\sqrt{4 \lambda_{2}+1}}{2 \lambda_{2}} \cdot \operatorname{Argsh}\left(\lambda_{2} \sqrt{\alpha}\right)\end{array}$ & $\frac{Q_{i}}{2 \pi \lambda_{i} h_{i} B} \cdot \operatorname{Argsh}\left(\lambda_{1} \sqrt{\alpha}\right)$ \\
\hline \multirow{2}{*}{$\begin{array}{l}1,5 \geq \lambda_{1}>0,7 \\
\lambda_{2}>1,5\end{array}$} & $\begin{array}{l}1,5 \geq \lambda_{1} \sqrt{\alpha}>0,7 \\
\lambda_{2} \sqrt{\alpha}>1,5\end{array}$ & $\frac{h_{1} Q_{2}}{2 \mathrm{nh}_{2} \mathrm{Q}_{1}}$ & $\frac{x}{\sqrt{4 x+1} \cdot \operatorname{Argsh}(n x)}$ & & $\begin{array}{l}\frac{k_{h}}{k_{1}}=\sqrt{\frac{\alpha\left(4 \lambda_{1}+1\right)}{4 \lambda_{1} \sqrt{\alpha+1}}} \\
\frac{k_{h}}{k_{2}}=\frac{\operatorname{Argsh}\left(\lambda_{2} \sqrt{\alpha}\right)}{\operatorname{Argsh} \lambda_{2}}\end{array}$ & $\begin{array}{l}\frac{Q_{1}}{\pi h_{1} B} \sqrt{\frac{\alpha}{4 \lambda_{1} \sqrt{\alpha+1}}}= \\
\frac{Q_{2}}{2 \pi \lambda_{2} h_{2} B} \cdot \operatorname{Argsh}\left(\lambda_{2} \sqrt{\alpha}\right)\end{array}$ \\
\hline & $\begin{array}{l}\lambda_{1} \sqrt{\alpha}>1,5 \\
\lambda_{2} \sqrt{\alpha}>1,5\end{array}$ & $\frac{h_{1} Q_{2}}{n h_{2} Q_{1}}$ & $\frac{\operatorname{Argsh} x}{\operatorname{Argsh}(n x)}$ & & $\begin{array}{l}\frac{k_{h}}{k_{1}}=\frac{\sqrt{4 \lambda_{1}}+1}{2 \lambda_{1}} \cdot \operatorname{Argsh}\left(\lambda_{1} \sqrt{\alpha}\right) \\
\frac{k_{h}}{k_{2}}=\frac{\operatorname{Argsh}\left(\lambda_{2} \sqrt{\alpha}\right)}{\operatorname{Argsh} \lambda_{2}}\end{array}$ & $\frac{Q_{i}}{2 \pi \lambda_{i} h_{i} B} \cdot \operatorname{Arg} \operatorname{sh}\left(\lambda_{i} \sqrt{\alpha}\right)$ \\
\hline
\end{tabular}

TABleauiv Essais Lefranc en milieu indéfini-Cavité initiale d'élancement nul.

\begin{tabular}{|c|c|c|c|c|c|c|}
\hline Milleu réel & $\begin{array}{l}\text { Milieu } \\
\text { transformé }\end{array}$ & $q$ & $f(x)$ & $\alpha$ & $\frac{k_{11}}{k_{2}}$ & $k_{p}$ \\
\hline $0,7 \geq \lambda>0,3$ & $1,5 \geq \lambda \sqrt{\alpha}>0,7$ & $\frac{2 h_{1} Q_{2}}{\pi h_{2} Q_{1}}$ & $\sqrt{4 x+1}$ & {$\left[\frac{q^{2}-1}{4 \lambda}\right]^{2}$} & $\sqrt{\frac{\alpha(4 \lambda+1)}{8 \lambda \sqrt{\alpha+2}}}$ & $\frac{Q_{2}}{\pi h_{2} B} \sqrt{\frac{\alpha}{4 \lambda \sqrt{\alpha+1}}}$ \\
\hline $\begin{array}{l}1,5 \geq \lambda>0,7 \\
\lambda \geq 1,5\end{array}$ & $1,5>\lambda \sqrt{\alpha}$ & $\frac{h_{1} Q_{2}}{\pi h_{2} Q_{1}}$ & $\frac{x}{\operatorname{Argsh} x}$ & $\left(\frac{x}{\lambda}\right)^{2}$ & $\begin{array}{l}\frac{\sqrt{4 \lambda+1}}{2 \lambda} \cdot \operatorname{Argsh}(\lambda \sqrt{\alpha}) \\
\frac{\operatorname{Argsh}(\lambda \sqrt{\alpha})}{\operatorname{Argsh} \lambda}\end{array}$ & $\frac{Q_{2}}{2 \pi \lambda h_{2} B} \operatorname{Argsh}(\lambda \sqrt{\alpha})$ \\
\hline
\end{tabular}

On devra vérifier que les valeurs de $\alpha$ et de $k_{h}$ données dans le tableau ci-dessus satisfont également aux relations: $\frac{k_{h}}{k_{1}}=\sqrt{\alpha}$ et $k_{h}=\frac{Q_{1} \sqrt{\alpha}}{2 h_{1} B}$. 


\section{Annexes}

\section{Annexe 1. Transformation d'un milieu homogène anisotrope en un milieu homogène isotrope}

On sait que l'équation de continuité qui régit l'écoulement en régime permanent dans un milieu homogène et isotrope est l'équation de Laplace :

$$
\frac{\partial^{2} h}{\partial x^{2}}+\frac{\partial^{2} h}{\partial y^{2}}+\frac{\partial^{2} h}{\partial z^{2}}=0
$$

où h est la charge hydraulique.

En milieu anisotrope caractérisé par un coefficient de perméabilité différent suivant chaque axe de coordomnée et que l'on désigne par $\mathrm{k}_{\mathrm{x}}, \mathrm{k}_{\mathrm{v}}$ et $\mathrm{k}_{\mathrm{z}}$ l'équation de continuité devient:

$$
k_{x} \frac{\partial^{2} h}{\partial x^{2}}+k_{y} \frac{\partial^{2} h}{\partial y^{2}}+k_{z} \frac{\partial^{2} h}{\partial z^{2}}=0
$$

Le problème consiste donc à transformer cette équation en équation de Laplace. Pour cela, on transforme le milieu géométrique réel en un milieu correspondant par l'affinité :

$$
x^{\prime}=\frac{x}{a} \quad y^{\prime}=\frac{y}{b} \quad z^{\prime}=\frac{z}{c}
$$

où $\mathrm{x}, \mathrm{y}$ et $\mathrm{z}$ sont les coordonnées d'un point $\mathrm{M}$ quelconque du milieu réel et $x^{\prime}, y^{\prime}$ et $z^{\prime}$, les coordonnées du transformé $\mathrm{M}^{\prime}$ de $\mathrm{M}$ dans le milieu correspondant, $\mathrm{a}, \mathrm{b}$ et $c$ étant des constantes arbitraires.

On a donc :

$$
\begin{aligned}
& \frac{\partial h}{\partial x}=\frac{\partial h}{d x^{\prime}} \cdot \frac{d x^{\prime}}{d x}=\frac{1}{a} \frac{\partial h}{\partial x^{\prime}} \text { etc. } \\
& \frac{\partial^{2} h}{\partial x^{2}}=\frac{1}{a^{2}} \frac{\partial^{2} h}{\partial x^{\prime 2}} \quad \text { etc. }
\end{aligned}
$$
vient :

En portant ces expressions dans l'équation (A.2), il

$$
\frac{k_{x}}{a^{2}} \frac{\partial^{2} h}{\partial x^{\prime 2}}+\frac{k_{y}}{b^{2}} \frac{\partial^{2} h}{\partial y^{\prime 2}}+\frac{k_{z}}{c^{2}} \frac{\partial^{2} h}{\partial z^{\prime 2}}=0
$$

Pour que cette équation soit une équation de Laplace, il faut et il suffit que :

$$
\frac{k_{x}}{a^{2}}=\frac{k_{y}}{b^{2}}=\frac{k_{z}}{c^{2}}=k
$$

on en déduit donc :

$$
\frac{1}{\mathrm{a}}=\sqrt{\frac{\mathrm{k}}{\mathrm{k}_{\mathrm{x}}}} \quad \frac{1}{\mathrm{~b}}=\sqrt{\frac{\mathrm{k}}{\mathrm{k}_{\mathrm{y}}}} \quad \frac{1}{\mathrm{c}}=\sqrt{\frac{\mathrm{k}}{\mathrm{k}_{z}}}
$$

où $\mathrm{k}$ est une constante arbitraire ayant la dimension d'un coefficient de perméabilité.

La transformation cherchée est donc :

$$
\begin{aligned}
& x^{\prime}=x \sqrt{\frac{k}{k_{x}}} \\
& y^{\prime}=y \sqrt{\frac{k}{k_{y}}} \\
& z^{\prime}=z \sqrt{\frac{k}{k_{z}}}
\end{aligned}
$$

Soit $\vec{V}(u, v, w)$ la vitesse de filtration dans le domaine réel où :

$$
\mathrm{u}=\frac{\mathrm{dx}}{\mathrm{dt}} \quad \mathrm{v}=\frac{\mathrm{dy}}{\mathrm{dt}} \quad \text { et } \quad \mathrm{w}=\frac{\mathrm{dz}}{\mathrm{dt}}
$$

Le débit dQ qui traverse un parallélépipède de côté $\mathrm{dx}$, dy et dz est alors :

$$
d Q=u d y d z+v d x d z+w d x d y
$$

Dans le domaine transformé, la vitesse de filtration est $\vec{V}^{\prime}\left(u^{\prime}, v^{\prime}, w^{\prime}\right)$ où :

$$
\mathrm{u}^{\prime}=\frac{\mathrm{dx}^{\prime}}{\mathrm{dt}}=\frac{1}{\mathrm{a}} \frac{\mathrm{dx}}{\mathrm{dt}}=\frac{\mathrm{u}}{\mathrm{a}} \quad \mathrm{v}^{\prime}=\frac{\mathrm{v}}{\mathrm{b}} \quad \text { et } \quad \mathrm{w}^{\prime}=\frac{\mathrm{w}}{\mathrm{c}}
$$

Le débit dQ' qui traverse un parallélépipède de côté $\mathrm{dx} \mathrm{x}^{\prime}, \mathrm{dy} \mathrm{y}^{\prime}$ et dz' est donc :

$$
\begin{aligned}
& d Q^{\prime}=u^{\prime} d y^{\prime} d z^{\prime}+v^{\prime} d x^{\prime} d z^{\prime}+w^{\prime} d x^{\prime} d y^{\prime} \\
& d Q^{\prime}=\frac{1}{a b c}(u d y d z+v d x d z+w d x d y)=\frac{d Q}{a b c}
\end{aligned}
$$

Ainsi donc, au débit $Q$ dans le milieu réel anisotrope, correspond dans le milieu transformé isotrope, un débit $Q^{\prime}$ tel que :

$$
Q^{\prime}=Q \sqrt{\frac{k^{3}}{k_{x} k_{y} k_{z}}}
$$

Puisque k est arbitraire, on peut lui attribuer n'importe quelle valeur et en particulier celles qui simplifient le problème. C'est ainsi par exemple que, si l'on souhaite que les débits restent inchangés dans les deux milieux, il suffira d'écrire $Q^{\prime}=Q$ d'où :

$$
\mathrm{k}=\sqrt[3]{\mathrm{k}_{\mathrm{x}} \mathrm{k}_{\mathrm{y}} \mathrm{k}_{\mathrm{z}}}
$$

soit encore si on admet que le milieu réel est horizontalement isotrope, c'est-à-dire si $k_{x}=k_{y}=k_{h}$ et $k_{z}=k_{v}$ :

$$
\mathrm{k}=\sqrt[3]{\mathrm{k}_{\mathrm{h}}^{2} \mathrm{k}_{\mathrm{v}}}
$$

Mais il est souvent plus intéressant de chercher à conserver les distances, soit horizontales, soit verticales. C'est ainsi que, toujours dans l'hypothèse où $k_{x}=$ $\mathrm{k}_{\mathrm{y}}=\mathrm{k}_{\mathrm{b}}$, on a :

- Conservation des distances horizontales

$$
\begin{aligned}
& k=k_{h} \\
& x^{\prime}=x \quad \text { et } \quad y^{\prime}=y \\
& z^{\prime}=z \sqrt{\frac{k_{h}}{k_{\mathrm{v}}}} \\
& Q^{\prime}=Q \sqrt{\frac{k_{h}}{k_{v}}}
\end{aligned}
$$

- Conservation des distances verticales :

$$
\begin{aligned}
& \mathrm{k}=\mathrm{k}_{\mathrm{v}} \\
& \mathrm{x}^{\prime}=\mathrm{x} \sqrt{\frac{\mathrm{k}_{\mathrm{v}}}{\mathrm{k}_{\mathrm{h}}}} \\
& \mathrm{y}^{\prime}=\mathrm{y} \sqrt{\frac{\mathrm{k}_{\mathrm{v}}}{\mathrm{k}_{\mathrm{h}}}} \\
& \mathrm{z}^{\prime}=\mathrm{z} \\
& \mathrm{Q}^{\prime}=\mathrm{Q} \frac{\mathrm{k}_{\mathrm{v}}}{\mathrm{k}_{\mathrm{h}}}
\end{aligned}
$$




\section{Annexe 2}

Table numérique 1 $\quad y(x, n)=\frac{\operatorname{Argsh} x}{\operatorname{Argsh}(n x)} \quad n \leq 5$

\begin{tabular}{|c|c|c|c|c|c|c|c|c|}
\hline$x$ & 1.5 & 2 & 2,5 & 3 & 3,5 & 4 & 4.5 & 5 \\
\hline 1,0 & 0,7376973 & 0,6105236 & 0,5350637 & 0,4846849 & 0,4483718 & 0,4207611 & 0,3989293 & 0,3811447 \\
\hline 1,5 & 0,7707364 & 0,6570241 & 0,5879090 & 0,5407765 & 0,5061860 & 0,4794819 & 0.4580915 & 0,4404713 \\
\hline 2,0 & 0,7938840 & 0,6891807 & 0,6242914 & 0,5793592 & 0,5459794 & 0,5199531 & 0,4989333 & 0,4814970 \\
\hline 2,5 & 0,8105556 & 0,7123352 & 0,6505477 & 0,6072819 & 0,5748594 & 0,5494025 & 0,5287239 & 0,5114875 \\
\hline 3,0 & 0,8230694 & 0,7297781 & 0,6704036 & 0,6284713 & 0,5968416 & 0,5718771 & 0,5515114 & 0,5344746 \\
\hline 3,5 & 0,8328179 & 0,7434307 & 0,6860075 & 0,6451792 & 0,6142234 & 0,5896906 & 0,5696100 & 0,5527644 \\
\hline 4,0 & 0.8406491 & 0,7544511 & 0,6986514 & 0,6587591 & 0,6283866 & 0,6042363 & 0,5844150 & 0,5677491 \\
\hline 4,5 & 0,8470996 & 0,7635702 & 0,7091505 & 0,6700667 & 0,6402062 & 0,6163976 & 0,5968127 & 0,5803145 \\
\hline 5,0 & 0,8525227 & 0,7712696 & 0,7180433 & 0,6796678 & 0,6502621 & 0,6267613 & 0,6073926 & 0,5910502 \\
\hline 5,5 & 0,8571598 & 0,7778790 & 0.7256990 & 0,6879517 & 0,6589538 & 0,6357321 & 0,6165619 & 0,6003645 \\
\hline 6,0 & 0,8611813 & 0.7836314 & 0,7323796 & 0,6951949 & 0,6665657 & 0,6435989 & 0,6246116 & 0,6085494 \\
\hline 6,5 & 0,8647108 & 0,7886969 & 0,7382762 & 0,7015997 & 0,6733063 & 0,6505734 & 0,6137555 & 0,6158195 \\
\hline 7,0 & 0,8678404 & 0,7932019 & 0,7435319 & 0.7073178 & 0,6793320 & 0,6568150 & 0,6381545 & 0,6223365 \\
\hline 7,5 & 0,8706399 & 0,7972430 & 0,7482557 & 0,7124650 & 0,6847627 & 0,6624458 & 0,6439321 & 0,6282249 \\
\hline 8,0 & 0,8731634 & 0,8008952 & 0,7525327 & 0,7171318 & 0,6896919 & 0,6675613 & 0,6491849 & 0,6335820 \\
\hline 8,5 & 0,8754535 & 0,8042175 & 0,7564300 & 0,7213897 & 0,6941939 & 0,6722373 & 0,6539898 & 0,6384853 \\
\hline 9,0 & 0,8775443 & 0,8072573 & 0,7600014 & 0,7252963 & 0,6983283 & 0,6765348 & 0,6584087 & 0,6429971 \\
\hline 9,5 & 0,8794631 & 0,8100529 & 0,7632908 & 0.7288983 & 0,7021437 & 0,6805036 & 0,6624921 & 0,6471686 \\
\hline 10,0 & 0,8812324 & 0,8126358 & 0,7663341 & 0,7322343 & 0,7056802 & 0,6841848 & 0,6662817 & 0,6510417 \\
\hline 15,0 & 0,8936610 & 0,8309208 & 0,7879967 & 0,7560794 & 0,7310420 & 0,7106555 & 0,6935939 & 0,6790110 \\
\hline 20,0 & 0,9010609 & 0,8419329 & 0,8011482 & 0,7706437 & 0,7466072 & 0,7269653 & 0,7104781 & 0,6963506 \\
\hline 25,0 & 0,9061430 & 0,8495534 & 0,8102976 & 0,7808168 & 0,7575141 & 0,7384239 & 0,7223663 & 0,7085827 \\
\hline 30,0 & 0,9099296 & 0,8552626 & 0,8171790 & 0,7884906 & 0,7657607 & 0,7471044 & 0,7313868 & 0,7178770 \\
\hline 35,0 & 0.9129035 & 0,8597659 & 0,8226231 & 0,7945756 & 0,7723118 & 0,7540103 & 0,7385725 & 0,7252888 \\
\hline 40,0 & 0,9153268 & 0,8634481 & 0,8270856 & 0,7995726 & 0,7776995 & 0,7596969 & 0,7444954 & 0,7314035 \\
\hline 45,0 & 0,9173559 & 0,8665403 & 0,8308407 & 0,8037840 & 0,7822457 & 0,7645001 & 0,7495025 & 0,7365767 \\
\hline 50,0 & 0,9190909 & 0,8691907 & 0,8340649 & 0,8074048 & 0,7861585 & 0,7686377 & 0,7538189 & 0,7410390 \\
\hline 55,0 & 0,9205992 & 0,8714997 & 0,8368780 & 0,8105674 & 0,7895793 & 0,7722578 & 0,7575979 & 0,7449479 \\
\hline 60,0 & 0,9219281 & 0,8735379 & 0,8393644 & 0.8133657 & 0,7926084 & 0,7754654 & 0,7609483 & 0.7484152 \\
\hline 65,0 & 0,9231120 & 0,8753568 & 0,8415859 & 0,8158680 & 0,7953192 & 0,7783377 & 0,7639498 & 0,7515227 \\
\hline 70,0 & 0,9241768 & 0,8769950 & 0,8435888 & 0,8181259 & 0,7977667 & 0,7809324 & 0,7666625 & 0,7543324 \\
\hline 75,0 & 0,9251419 & 0,8784820 & 0,8454085 & 0,8201789 & 0,7999933 & 0,7832941 & 0,7691326 & 0.7568917 \\
\hline 80,0 & 0,9260228 & 0,8798408 & 0,8470730 & 0,8220578 & 0,8020324 & 0,7854579 & 0,7713965 & 0.7592381 \\
\hline 85,0 & 0,9268317 & 0,8810900 & 0,8486042 & 0,8237875 & 0,8039104 & 0,7874514 & 0,7734831 & 0,7614013 \\
\hline 90,0 & 0,9275783 & 0,8822442 & 0,8500201 & 0.8253879 & 0,8056487 & 0,7892974 & 0,7754159 & 0,7634057 \\
\hline 95,0 & 0,9282707 & 0,8833156 & 0,8513354 & 0,8268752 & 0,8072649 & 0,7910144 & 0,7772140 & 0,7652709 \\
\hline 100,0 & 0,9289155 & 0,8843141 & 0,8525620 & 0,8282630 & 0,8087736 & 0,7926176 & 0,7788935 & 0,7670135 \\
\hline
\end{tabular}


Table numérique 1 suite $\quad y(x, n)=\frac{\operatorname{Argsh} x}{\operatorname{Argsh}(n x)} \quad n>5$

\begin{tabular}{|c|c|c|c|c|c|c|c|c|}
\hline$\times$ & 6 & 7 & 8 & 9 & 10 & 12 & 14 & 16 \\
\hline 1,0 & 0,3537125 & 0,3333333 & 0,3174437 & 0,3046016 & 0,2939653 & 0,2771802 & 0,2644006 & 0,2542391 \\
\hline 1,5 & 0,4129208 & 0,3921393 & 0,3757371 & 0,3623563 & 0,3511627 & 0,3333333 & 0,3196061 & 0,3085940 \\
\hline 2,0 & 0,4540041 & 0,4330719 & 0,4164280 & 0,4027675 & 0,3912817 & 0,3728749 & 0,3586075 & 0,3471006 \\
\hline 2,5 & 0,4841513 & 0,4632046 & 0,4464641 & 0,4326671 & 0,4210259 & 0,4022914 & 0,3877025 & 0,3758928 \\
\hline 3,0 & 0,5073380 & 0,4864451 & 0,4696844 & 0,4558279 & 0,4441060 & 0,4251827 & 0,4103957 & 0,3983928 \\
\hline 3,5 & 0,5258417 & 0,5050362 & 0,4882964 & 0,4744236 & 0,4626641 & 0,4436332 & 0,4287219 & 0.4165917 \\
\hline 4,0 & 0,5410408 & 0,5203388 & 0,5036423 & 0,4897783 & 0.4780067 & 0,4589183 & 0,4439287 & 0,4317133 \\
\hline 4,5 & 0,5538147 & 0,5332227 & 0,5165818 & 0,5027413 & 0,4909735 & 0,4718593 & 0,4568218 & 0,4445491 \\
\hline 5,0 & 0,5647501 & 0,5442697 & 0,5276910 & 0,5138828 & 0,5021287 & 0,4830097 & 0,4679445 & 0,4556338 \\
\hline 5,5 & 0,5742543 & 0,5538843 & 0,5373707 & 0,5236002 & 0,5118662 & 0,4927562 & 0,4776775 & 0,4653421 \\
\hline 6,0 & 0,5826191 & 0,5623568 & 0,5459094 & 0,5321795 & 0,5204696 & 0,5013781 & 0,4862959 & 0.4739456 \\
\hline 6,5 & 0,5900596 & 0,5699016 & 0,5535201 & 0,5398323 & 0,5281490 & 0,5090826 & 0,4940039 & 0,4816459 \\
\hline 7.0 & 0,5967379 & 0,5766804 & 0,5603638 & 0,5467188 & 0,5350637 & 0,5160267 & 0,5009569 & 0,4885964 \\
\hline 7,5 & 0,6027790 & 0,5828181 & 0,5665651 & 0,5529629 & 0,5413368 & 0,5223323 & 0,5072750 & 0,4949161 \\
\hline 8,0 & 0,6082810 & 0,5884129 & 0,5722218 & 0,5586619 & 0,5470652 & 0,5280952 & 0,5130532 & 0,5006990 \\
\hline 8,5 & 0,6133218 & 0,5935427 & 0,5774117 & 0,5638936 & 0,5523263 & 0,5333920 & 0,5183674 & 0,5060202 \\
\hline 9,0 & 0,6179645 & 0,5982708 & 0,5821981 & 0,5687209 & 0,5571829 & 0,5382851 & 0,5232793 & 0,5109408 \\
\hline 9,5 & 0,6222606 & 0,6026489 & 0,5866326 & 0,5731954 & 0,5616864 & 0,5428255 & 0,5278395 & 0,5155112 \\
\hline 10,0 & 0,6262527 & 0,6067197 & 0,5907581 & 0,5773599 & 0,5658794 & 0,5470555 & 0,5320902 & 0,5197731 \\
\hline 15,0 & 0,6551733 & 0,6362865 & 0,6207845 & 0,6077245 & 0,5964989 & 0,5780228 & 0,5632715 & 0,5510888 \\
\hline 20,0 & 0,6731865 & 0,6547708 & 0,6396138 & 0,6268151 & 0.6157927 & 0,5976076 & 0,5830498 & 0,5710006 \\
\hline 25,0 & 0,6859334 & 0,6678836 & 0,6529987 & 0,6404094 & 0,6295522 & 0,6116092 & 0,5972178 & 0,5852878 \\
\hline 30,0 & 0,6956413 & 0,6778884 & 0,6632266 & 0,6508106 & 0,6400915 & 0,6223537 & 0,6081059 & 0,5962810 \\
\hline 35,0 & 0,7033967 & 0,6859925 & 0,6714190 & 0,6591502 & 0,6485492 & 0,6309884 & 0,6168662 & 0,6051343 \\
\hline 40,0 & 0,7098043 & 0,6925133 & 0,6782021 & 0,6660608 & 0,6555627 & 0,6381571 & 0,6241461 & 0,6124973 \\
\hline 45,0 & 0,7152319 & 0,6981270 & 0,6839580 & 0,6719291 & 0,6615218 & 0,6442542 & 0.6303427 & 0,6187687 \\
\hline 50,0 & 0,7199185 & 0,7029785 & 0,6889359 & 0,6770071 & 0,6666810 & 0,6495374 & 0,6357158 & 0,6242099 \\
\hline 55,0 & 0,7240277 & 0,7072353 & 0,6933062 & 0,6814676 & 0,6712150 & 0,6541836 & 0,6404440 & 0,6290003 \\
\hline 60,0 & 0,7276755 & 0,7110165 & 0,6971905 & 0,6854338 & 0,6752481 & 0,6583194 & 0,6446549 & 0,6332685 \\
\hline 65,0 & 0,7309472 & 0,7144100 & 0,7006780 & 0,6889963 & 0,6788720 & 0,6620377 & 0,6484425 & 0,6371093 \\
\hline 70,0 & 0,7339072 & 0,7174817 & 0,7038362 & 0,6922237 & 0,6821560 & 0,6654091 & 0,6518782 & 0,6405944 \\
\hline 75,0 & 0,7366051 & 0,7202827 & 0,7067172 & 0,6951688 & 0,6851536 & 0,6684880 & 0,6550171 & 0,6437794 \\
\hline 80.0 & 0,7390799 & 0,7228531 & 0,7093620 & 0,6978733 & 0,6879071 & 0,6713173 & 0,6579026 & 0,6467082 \\
\hline 85,0 & 0,7413626 & 0,7252251 & 0,7118034 & 0,7003705 & 0,6904501 & 0,6739315 & 0,6605995 & 0,6494160 \\
\hline 90,0 & 0,7434786 & 0,7274246 & 0,7140681 & 0,7026874 & 0,6928101 & 0,6763584 & 0,6630462 & 0,6519312 \\
\hline 95,0 & 0,7454486 & 0,7294730 & 0,7161777 & 0,7048464 & 0,6950097 & 0,6786211 & 0,6653560 & 0,6542774 \\
\hline 100.0 & 0,7472898 & 0,7313881 & 0,7181506 & 0,7068658 & 0,6970675 & 0,6807388 & 0,6675182 & 0,6564743 \\
\hline
\end{tabular}


Table numérique 2 $\quad \frac{k_{h}}{k}=\frac{\operatorname{Argsh}(\lambda \sqrt{\alpha})}{\operatorname{Argsh} \lambda}$

\begin{tabular}{l|l|l|l|l|l|l|l|l|}
\hline$\alpha$ & 1,5 & 2 & 2,5 & 3 & 3,5 & 4 & 5 \\
\hline 5 & 1,61107 & 1,52621 & 1,47038 & 1,43089 & 1,40136 & 1,37836 & 1,34460 & 1,26773 \\
10 & 1,89229 & 1,76206 & 1,67840 & 1,61996 & 1,57664 & 1,54307 & 1.49404 & 1,38324 \\
15 & 2,05896 & 1,90108 & 1,80067 & 1,73095 & 1,67943 & 1,63961 & 1.58157 & 1,45083 \\
20 & 2,17783 & 2,00000 & 1,88759 & 1,80979 & 1,75243 & 1,70815 & 1,64370 & 1,49880 \\
25 & 2,27030 & 2,07686 & 1,95508 & 1,87100 & 1,80909 & 1.76134 & 1,69190 & 1,53600 \\
30 & 2,34598 & 2,13972 & 2,01026 & 1,92103 & 1,85540 & 1,80481 & 1,73130 & 1,56640 \\
35 & 2,41006 & 2,19290 & 2,05694 & 1,96334 & 1,89456 & 1,84157 & 1,76461 & 1,59210 \\
40 & 2,46561 & 2,23900 & 2,09738 & 2,00000 & 1,92848 & 1,87342 & 1,79346 & 1,61437 \\
45 & 2,51464 & 2,27967 & 2,13307 & 2,03234 & 1,95841 & 1,90151 & 1,81892 & 1,63401 \\
50 & 2,55853 & 2,31606 & 2,16500 & 2,06128 & 1,98519 & 1,92664 & 1,84169 & 1,65158 \\
55 & 2,59825 & 2,34900 & 2,19388 & 2,08746 & 2,00941 & 1,94938 & 1,82229 & 1,66747 \\
60 & 2,63452 & 2,37907 & 2,22026 & 2,11136 & 2,03153 & 1,97014 & 1,88110 & 1,68198 \\
65 & 2,66790 & 2,40674 & 2,24452 & 2,13335 & 2,05188 & 1,98923 & 1,89840 & 1,69533 \\
70 & 2,69881 & 2,43235 & 2,26699 & 2,15371 & 2,07072 & 2,00692 & 1,91442 & 1,70768 \\
75 & 2,72760 & 2,45621 & 2,28791 & 2,17266 & 2,08826 & 2,02338 & 1,92933 & 1,71919 \\
80 & 2,75453 & 2,47853 & 2,30748 & 2,19040 & 2,10466 & 2,03878 & 1,94328 & 1,72995 \\
85 & 2,77983 & 2,49949 & 2,32586 & 2,20706 & 2,12008 & 2,05324 & 1,95639 & 1,74006 \\
90 & 2,80369 & 2,51926 & 2,34320 & 2,22276 & 2,13461 & 2,06688 & 1,96874 & 1,74959 \\
95 & 2,82627 & 2,53796 & 2,35959 & 2,23762 & 2,14835 & 2,07978 & 1,98043 & 1,75861 \\
100,0 & 2,84768 & 2,55570 & 2,37515 & 2,25171 & 2,16140 & 2,09202 & 1,99152 & 1,76716 \\
\hline
\end{tabular}

Table numérique $3 \quad q=\frac{x}{\sqrt{4 x+1 \times \operatorname{Argsh}(n x)}}$

\begin{tabular}{|c|c|c|c|c|c|c|c|c|c|c|}
\hline & 1.5 & 2.0 & 2,5 & 3,0 & 3,5 & 4,0 & 4,5 & 5,0 & 10.0 & 15.0 \\
\hline 1,0 & 0,37431 & 0,30978 & 0,27149 & 0,24593 & 0,22751 & 0,21350 & 0,20242 & 0,19339 & 0,14916 & 0,13144 \\
\hline 1,5 & 0,36573 & 0,31178 & 0,27898 & 0,25661 & 0,24020 & 0,22753 & 0,21738 & 0,20902 & 0,16664 & 0,14892 \\
\hline 2,0 & 0,36661 & 0,31826 & 0,28830 & 0,26755 & 0,25213 & 0,24011 & 0,23041 & 0,22235 & 0,18069 & 0,16282 \\
\hline 2,5 & 0,37091 & 0,32597 & 0,29769 & 0,27789 & 0,26306 & 0,25141 & 0,24195 & 0,23406 & 0,19266 & 0,17458 \\
\hline 3,0 & 0,37660 & 0,33392 & 0,30675 & 0,28756 & 0,27309 & 0,26167 & 0,25235 & 0,24455 & 0,20321 & 0,18490 \\
\hline 3,5 & 0,38287 & 0,34178 & 0,31538 & 0,29661 & 0,28238 & 0,27110 & 0,26187 & 0,25412 & 0,21270 & 0.19417 \\
\hline 4,0 & 0,38934 & 0,34942 & 0,32357 & 0,30510 & 0,29103 & 0,27985 & 0,27067 & 0,26295 & 0,22138 & 0,20264 \\
\hline 4,5 & 0,39583 & 0,35680 & 0,33137 & 0,31310 & 0,29915 & 0,28803 & 0,27888 & 0,27117 & 0,22942 & 0,21046 \\
\hline 5,0 & 0,40225 & 0,36391 & 0,33880 & 0,32069 & 0,30682 & 0,29573 & 0,28659 & 0,27888 & 0,23692 & 0,21775 \\
\hline 5,5 & 0,40856 & 0,37077 & 0,34590 & 0,32791 & 0,31409 & 0,30302 & 0,29388 & 0,28616 & 0,24398 & 0,22461 \\
\hline 6,0 & 0,41473 & 0,37738 & 0,35270 & 0,33479 & 0,32101 & 0,30995 & 0,30080 & 0,29307 & 0,25065 & 0,23108 \\
\hline 6,5 & 0,42076 & 0,38377 & 0,35924 & 0,34139 & 0,32762 & 0,31656 & 0,30740 & 0,29965 & 0,25699 & 0,23723 \\
\hline 7,0 & 0,42664 & 0,38994 & 0,36553 & 0,34772 & 0,33396 & 0,32289 & 0,31372 & 0,30594 & 0,26304 & 0,24310 \\
\hline 7,5 & 0,43237 & 0,39592 & 0,37159 & 0,35382 & 0,34006 & 0,32898 & 0,31978 & 0,31198 & 0,26883 & 0,24871 \\
\hline 8.0 & 0,43796 & 0,40171 & 0,37745 & 0,35970 & 0,34594 & 0,33483 & 0,32562 & 0,31779 & 0,27440 & 0,25410 \\
\hline 8,5 & 0,44342 & 0,40734 & 0,38313 & 0,36538 & 0,35161 & 0,34049 & 0,33124 & 0,32339 & 0,27975 & 0,25928 \\
\hline 9,0 & 0,44874 & 0,41280 & 0,38863 & 0,37089 & 0,35710 & 0,34595 & 0,33668 & 0,32880 & 0,28492 & 0,26429 \\
\hline 9,5 & 0,45394 & 0,41811 & 0,39398 & 0,37623 & 0,36242 & 0,35125 & 0,34195 & 0,33404 & 0,28992 & 0,26912 \\
\hline 10,0 & 0,45902 & 0,42329 & 0,39917 & 0,38141 & 0,36758 & 0,35638 & 0,34706 & 0,33912 & 0,29476 & 0,27381 \\
\hline 15,0 & 0,50446 & 0,46904 & 0,44481 & 0,42680 & 0,41266 & 0,40115 & 0,39152 & 0,38329 & 0,33672 & 0,31437 \\
\hline 20,0 & 0.54272 & 0,50710 & 0,48254 & 0,46417 & 0,44969 & 0,43786 & 0,42793 & 0,41942 & 0,37090 & 0,34739 \\
\hline 25,0 & 0,57614 & 0,54016 & 0,51520 & 0,49646 & 0,48164 & 0.46950 & 0,45929 & 0,45053 & 0,40028 & 0,37577 \\
\hline 30,0 & 0,60607 & 0,56966 & 0,54429 & 0,52518 & 0,51004 & 0,49762 & 0,48715 & 0,47815 & 0,42634 & 0,40093 \\
\hline 35,0 & 0,63333 & 0,59646 & 0,57069 & 0,55124 & 0,53579 & 0,52309 & 0,51238 & 0,50317 & 0,44993 & 0,42371 \\
\hline 40,0 & 0,65847 & 0,62114 & 0,59499 & 0,57519 & 0,55946 & 0,54651 & 0,53557 & 0,52615 & 0,47160 & 0,44463 \\
\hline 45,0 & 0,68188 & 0,64410 & 0,61757 & 0,59746 & 0,58145 & 0,56826 & 0,55711 & 0,54750 & 0,49171 & 0,46405 \\
\hline 50,0 & 0,70384 & 0,66563 & 0,63873 & 0,61831 & 0,60204 & 0,58862 & 0,57728 & 0,56749 & 0,51055 & 0,48224 \\
\hline 55,0 & 0,72458 & 0,68594 & 0,65869 & 0,63798 & 0,62146 & 0,60783 & 0,59629 & 0,58633 & 0,52830 & 0,49938 \\
\hline 60,0 & 0,74426 & 0,70520 & 0,67761 & 0,65662 & 0,63986 & 0,62602 & 0,61430 & 0,60419 & 0,54512 & 0,51563 \\
\hline 65,0 & 0,76302 & 0,72354 & 0,69563 & 0,67437 & 0,65739 & 0,64335 & 0,63146 & 0,62118 & 0,56113 & 0.53110 \\
\hline 70,0 & 0,78095 & 0,74108 & 0,71285 & 0,69134 & 0,67413 & 0,65991 & 0,64785 & 0,63743 & 0,57644 & 0,54588 \\
\hline 75,0 & 0,79816 & 0,75790 & 0,72937 & 0,70760 & 0,69019 & 0,67578 & 0,66356 & 0,65300 & 0,59111 & 0,56006 \\
\hline 80,0 & 0,81471 & 0,77408 & 0,74525 & 0,72325 & 0,70563 & 0,69104 & 0,67867 & 0,66798 & 0,60522 & 0,57369 \\
\hline 85,0 & 0,83068 & 0,78968 & 0,76056 & 0,73832 & 0,72051 & 0,70576 & 0,69324 & 0,68241 & 0,61882 & 0,58683 \\
\hline 90,0 & 0,84610 & 0,80475 & 0,77536 & 0,75289 & 0,73488 & 0,71997 & 0,70730 & 0,69635 & 0,63195 & 0,59952 \\
\hline 95,0 & 0,86103 & 0,81934 & 0,78967 & 0,76698 & 0,74879 & 0,73372 & 0,72092 & 0,70984 & 0,64467 & 0,61181 \\
\hline 100,0 & 0,87552 & 0,83348 & 0,80355 & 0,78065 & 0,76228 & 0,74705 & 0,73412 & 0,72292 & 0,65700 & 0,62372 \\
\hline
\end{tabular}


Table numérique $4 \quad q=\frac{x}{\operatorname{Argsh} x}$

\begin{tabular}{l|c|c|c|}
$\mathrm{x}$ & $\mathrm{q}$ & $\mathrm{x}$ & $\mathrm{q}$ \\
\hline 1,5 & 1,25548 & 15 & 4,40877 \\
2,0 & 1,38539 & 20 & 5,42078 \\
2,5 & 1,51770 & 25 & 6,38990 \\
3,0 & 1,64976 & 30 & 7,32668 \\
3,5 & 1,78052 & 35 & 8,23782 \\
4,0 & 1,90957 & 40 & 9,12787 \\
4,5 & 2,03680 & 45 & 10,00015 \\
5,0 & 2,16222 & 50 & 10,85713 \\
5,5 & 2,28590 & 55 & 11,70073 \\
6,0 & 2,40792 & 60 & 12,53248 \\
6,5 & 2,52838 & 65 & 13,35362 \\
7,0 & 2,64738 & 70 & 14,16518 \\
7,5 & 2,76501 & 75 & 14,96803 \\
8,0 & 2,88135 & 80 & 15,76289 \\
8,5 & 2,99649 & 85 & 16,55038 \\
9,0 & 3,11048 & 90 & 17,33106 \\
9,5 & 3,22340 & 95 & 18,10541 \\
10,0 & 3,33531 & 100 & 18,87383 \\
\hline
\end{tabular}

\section{Bibliographie}

[1] Cambefort H. - Introduction à la gêotechnique, Eyrolles, 1971.

[2] Cassan M. - $\alpha$ Filtration dans les cavités souterraines 》. Annales de ITTBTP, avrilmai 1979.

[3] Cassan M. - Les essais d'eau dans la reconnaissance des sols, Eyrolles, 1980.
[4] Cassan M. - Aide-mèmoire d'hydraulique souterraine, Presses des Ponts et Chaussées, 1993, $2^{\circ}$ édition.

[5] Mandel J. - $\alpha$ Note sur le calcul des filtrations x. Annales des Ponts et Chaussées, 1939.
[6] Schneebeli G. $-\alpha$ La mesure in situ de la perméabilité d'un terrain $x$. Congrès de la Société hydrotechnique de France, Alger, 1954

[7] Schneebeli G. - Hydraulique souterraine, Eyrolles, 1966 